\title{
Cardiac C-Arm CT: A Unified Framework for Motion Estimation and Dynamic CT
}

\author{
Marcus Prümmer*, Joachim Hornegger, Guenter Lauritsch, Lars Wigström, Erin Girard-Hughes, and \\ Rebecca Fahrig
}

\begin{abstract}
Generating 3-D images of the heart during interventional procedures is a significant challenge. In addition to real time fluoroscopy, angiographic $\mathrm{C}$-arm systems can also now be used to generate 3-D/4-D CT images on the same system. One protocol for cardiac CT uses ECG triggered multisweep scans. A 3-D volume of the heart at a particular cardiac phase is then reconstructed by applying Feldkamp (FDK) reconstruction to the projection images with retrospective ECG gating. In this work we introduce a unified framework for heart motion estimation and dynamic cone-beam reconstruction using motion corrections. The benefits of motion correction are 1) increased temporal and spatial resolution by removing cardiac motion which may still exist in the ECG gated data sets and 2) increased signal-to-noise ratio (SNR) by using more projection data than is used in standard ECG gated methods. Three signal-enhanced reconstruction methods are introduced that make use of all of the acquired projection data to generate a 3-D reconstruction of the desired cardiac phase. The first averages all motion corrected back-projections; the second and third perform a weighted averaging according to 1) intensity variations and 2) temporal distance relative to a time resolved and motion corrected reference FDK reconstruction. In a comparison study seven methods are compared: nongated FDK, ECG-gated FDK, ECG-gated, and motion corrected FDK, the three signal-enhanced approaches, and temporally aligned and averaged ECG-gated FDK reconstructions. The quality measures used for comparison are spatial resolution and SNR. Evaluation is performed using phantom data and animal models. We show that data driven and subject-specific motion estimation combined with motion correction can decrease motion-related blurring substantially. Furthermore, SNR can be increased by up to $70 \%$ while maintaining spatial resolution at the same level as is provided by the ECG-gated FDK. The presented framework provides excellent image quality for cardiac C-arm CT.
\end{abstract}

Manuscript received December 22, 2008; revised May 29, 2009. Current version published October 28, 2009. This work was supported in part by Siemens AG, Healthcare Sector, Forchheim, Germany, in part by the National Institutes of Health under Grant R01 EB 003524, in part by the Lucas Foundation, HipGraphics, Towson, MD, and in part by the Bavaria California Technology Center $(\mathrm{BaCaTec})$. The concepts and information presented in this paper are based on research and are not commercially available. Asterisk indicates corresponding author.

*M. Prümmer is with the Chair of Pattern Recognition, FA-University Erlangen-Nuremberg, 91054 Nuremberg, Germany (e-mail: pruemmer@informatik.uni-erlangen.de).

J. Hornegger is with the Chair of Pattern Recognition, FA-University Erlangen-Nuremberg, 91054 Nuremberg, Germany (e-mail: hornegger@informatik.uni-erlangen.de).

G. Lauritsch is with the Siemens AG, Healthcare Sector, 91301 Forchheim, Germany (e-mail: guenter.lauritsch@siemens.com).

L. Wigström, E. Girard-Hughes, and R. Fahrig are with the Department of Radiology, Stanford University, Stanford, CA 94305 USA (e-mail: egirard@ stanford.edu; fahrig@stanford.edu; larsw@stanford.edu).

Color versions of one or more of the figures in this paper are available online at http://ieeexplore.ieee.org.

Digital Object Identifier 10.1109/TMI.2009.2025499
Index Terms-Cardiac C-arm CT, dynamic object reconstruction in CT, heart motion estimation, retrospective motion correction, signal-to-noise ratio (SNR) enhancement.

\section{CARDiAC IMAGING}

\section{A. Medical Application}

A CCESS to intraprocedural 3-D images in the interventional suite is becoming more important as minimally invasive cardiac procedures increase in complexity. Retrospectively ECG-gated cardiac C-arm CT has recently been developed [2], allowing a single $\mathrm{C}$-arm imaging system to provide both real-time fluoroscopy and 3-D volume CT images of the heart during a procedure. The imaging protocol for this $3-\mathrm{D}$ volume imaging approach is to acquire 2-D projection images during sequential forward and backward sweeps around the object while simultaneously recording the ECG signal. A 3-D volume reconstruction of a particular cardiac phase is accomplished by choosing the projection at each angle of the set of projections that is closest to the phase of interest, and then using the standard Feldkamp reconstruction algorithm (FDK) [1] to generate the 3-D volume. Current 3-D image quality, as defined by signal-to-noise ratio (SNR) and motion-related blurring (MRB), is determined by the total imaging time (which must be within a single breath hold) the time per sweep, the detector readout rate and the dose per projection. These parameters determine the number of projections (and therefore the dose) and the temporal spread of the projection data that contribute to a single reconstructed volume at a given cardiac phase. Note that the slower rotation speed of the C-arm leads to a temporal spread of the ECG-gated projections that is much higher than that produced by a clinical CT scanner. It is likely that a preoperative clinical CT scan will not be obtained if intraoperative $\mathrm{C}$-arm CT is used, in order to minimize patient exposure.

Improvement of image quality for these typically view-starved 3-D reconstructions (e.g., $\sim 200$ views per volume as compared to $\sim 1000$ for clinical CT) may be particularly important if there is a need to use automatic segmentation and/or 2-D/3-D image registration algorithms during the intervention. One approach to improve image quality and to increase the dose efficiency of the ECG-gated C-arm CT imaging protocol is to use all of the projection data acquired to produce a single volume at the cardiac phase of interest. Such an approach increases the SNR of the reconstructed volume, but requires 
knowledge of and correction for the motion of the object in order to limit MRB. Many methods for motion estimation and for motion correction for both respiratory and cardiac motion in 3-D reconstructed images have been developed. A summary of the current state of the art is presented below. In general, the motion is first modeled via a mathematical model, using dense deformation fields or using spline models. In the second step, a reconstruction method that takes the motion into consideration is applied to improve the quality of the reconstruction. This combination of motion estimation and dynamic object reconstruction reduces $\mathrm{MRB}$ and can improve image quality in cardiac C-arm CT.

\section{B. Contributions to Cardiac C-Arm CT}

In this paper, we present new work demonstrating SNR improvement using an estimated dense deformation field in combination with a modified FDK algorithm for use with the ECG-gated C-arm CT imaging protocol. We follow the two-step process as outlined above, with some key refinements. First, the motion vector field (MVF) mapping the reconstructed cardiac phases to a reference phase is calculated using a multilevel 3-D/3-D registration approach that has been runtime optimized to provide a fast estimate of the MVF suitable for use in the clinic. We then concatenate these dense deformation fields to generate a time-continuous 4-D MVF using interpolation between cardiac phases, so that the trajectory of each voxel throughout the whole cardiac cycle is known. To reconstruct the corrected volume, each projection is backprojected along a curved path, with the path determined by the voxel being reconstructed, the phase at which the projection was acquired, and the estimated MVF. We carry out motion correction in the projection space, which maximizes the resulting image quality for a given accuracy of the MVF.

Since subject-specific heart motion encoded in the MVF can only be estimated approximately and nonrigid heart motion cannot be corrected exactly by current dynamic FDK-like algorithms, a trade-off exists between spatial resolution and SNR, depending on the projection data used for reconstruction. In this work we present two new weighting methods to combine all of the acquired projection data from a multisweep protocol (see Lauritsch et al. [2]) into a single reconstructed volume: weighting by cardiac phase variance and weighting by intensity variance. All weighting schemes are based on the combination of several short-scan motion compensated FDK-like reconstructions. The resulting motion-corrected image quality is compared to uncorrected FDK-like reconstructions and also compared to the current state-of-the-art ECG-gated Feldkamp reconstruction, in the following denoted as EG-FDK. Image quality is evaluated and compared by measuring the edge response function versus SNR for all of the reconstruction methods. In summary the major contributions of this paper are combined motion estimation and correction, SNR enhancement, and further image quality improvements.

\section{Motion Vector Field Estimation}

Initial studies using animal models by Prümmer et al. [5] have shown that a 4-D MVF can be derived for this application by computation of a subject-specific series of 3-D MVFs using a variational nonrigid registration approach. The estimation of the MVFs is based on a time series of EG-FDK reconstructions. A comprehensive review of variational nonrigid registration can be found in [21], including details of distance measures such as sum of squared differences, correlation based measures and mutual information as well as smoothness regularizations for the deformation. Most of the registration algorithms proposed in the literature provide a nonsymmetric motion estimation. The motion is computed starting from the fixed object (reference) towards the moving object. However, as introduced by Han $e t$ al. [20], a symmetric motion estimation that provides a bijective mapping between the aligned volumes is desired. Han introduced a regularized Mumford-Shah Model that provides a one-to-one edge matching of the aligned objects. In comparison to injective mappings such an approach provides the transformation over time in both directions. Therefore no spatial regridding of the dense deformation field is required to transform the volume to another cardiac phase in both time directions. However, a bijective mapping is computationally more expensive and it is not required for our application.

A general review of 3-D modeling for functional analysis of cardiac images in different modalities is given by Frangi et al. [8]. A 4-D image registration method for consistent estimation of cardiac motion from MRI image sequences was proposed by Shen et al. [11]. Within this 4-D registration framework, all 3-D cardiac images obtained at different time-points are registered simultaneously and the motion estimation is forced to be spatiotemporally smooth. This smoothness constraint overcomes the potential limitations of those methods that estimate cardiac deformation sequentially from one frame to another instead of treating the entire set of images as a 4-D volume.

Taguchi et al. [10] presented a method that estimates the 2-D components of the MVF from a time sequence of 2-D cardiac CT slices. Taguchi et al. [9] has also proposed an iterative approach repeating the following four steps until the difference between two projection data sets falls below a certain criterion: 1) estimate or update the cardiac motion vectors, 2) reconstruct the time-resolved 4-D dynamic image volume using the motion vectors, 3) calculate forward projections from the current 4-D images, and 4) compare them with the measured projection data.

We choose a fast and parallel 3-D/3-D nonrigid multilevel registration method to deal with larger deformations and avoid error propagation over time. No prior knowledge for motion modeling is used, providing a purely subject-specific motion estimation so that the anatomical structure of contrast-filled ventricles can be optimally aligned. This is especially important for dynamic CT reconstruction of an individual subject.

\section{Dynamic 3-D Reconstruction}

Many motion correction methods for respiratory and cardiac motion have been proposed in the literature. Most of the methods consist of two steps. First, the motion is modeled via a mathematical model, dense deformation fields, or spline models. Second, a reconstruction method incorporates the motion during reconstruction. Here one can distinguish between correcting the motion in the projection space or in the image space of the reconstructed volume or slice. In addition, there 
are different classes of object motion, such as linear, affine or generalized nonrigid motion.

Desbat et al. [23] presented a general work for $2-\mathrm{D}+t$ dynamic tomography and also proposed a generalization to 3-D cone-beam; their scheme compensates analytically within filtered backprojection for object deformations that are affine in time and along a line (ray). Taguchi et al. [24] introduced a method for motion compensated reconstruction using derivative backprojection filtering that corrects for locally affine transformations. These classes of deformations, however, do not include arbitrary nonrigid deformations like complex cardiac motion.

Blondel et al. [12] introduced a method that consists of three steps: 1) 3-D reconstruction of coronary artery centerlines, including respiratory motion compensation, 2) computation of the 4-D coronary artery motion, and 3) 3-D tomographic reconstruction of coronary arteries, with compensation for respiratory and cardiac motion. For the motion compensated reconstruction a dynamic projector model combined with an iterative ART method is used.

Li et al. [7] presented a first version of motion compensated reconstruction. They used a time-dependent transformation of 3-D filtered backprojections to incorporate a patient-specific motion model, and extended the algorithm to 3-D for cone-beam CT. It has also been shown that given a motion field of a dynamic (nonrigid) moving object, a motion corrected (dynamic) FDK-like reconstruction can be performed (Schäfer et al. [3] and [4]). The dynamic reconstruction is performed by dynamically adapting the geometry used for filtered backprojection according to the MVFs. These methods can deal with arbitrary nonrigid cardiac motion, but the filtering and redundancy weighting is still approximate.

Iterative motion estimation and reconstruction methods (e.g., [9]) are time consuming. This is especially true when the energy functional contains a combination of 3-D/4-D image data and 2-D projection images. Methods where the motion is only estimated in 2-D projection space are limited because 3-D motion cannot be uniquely measured in sinogram space. Furthermore cardiac motion lies in the generalized deformation class of nonrigid motion. In this work we therefore use noniterative, combined motion estimation and correction in $3-\mathrm{D}+t$ using an approximate, but fast dynamic filtered backprojection approach. The motion correction method is based on the work of Li et al. [7] and has been optimized for the recently introduced multisweep C-arm acquisition protocol [2].

\section{E. SNR Enhanced Reconstruction}

An approach for respiratory motion compensation and SNR enhancement was introduced by Li et al. [6]. The 3-D CT images at different phases are registered to the same phase via a deformable model. A regularization term combining temporal and spatial neighbors is proposed and thus dose reduction can be achieved. A second method [22] introduced for 4-D conebeam CT (4DCBCT) correlates the data in different respiratory phase bins and integrates the internal respiratory motion into the 4DCBCT reconstruction. Each filtered backprojection is deformed by a time-dependent transformation to correct for motion. This approach is similar to our method, but we address the problem of cardiac motion, which is more complex because

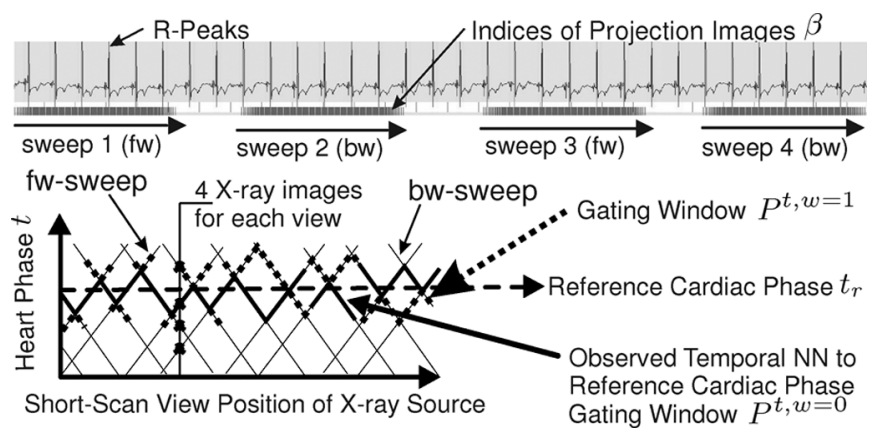

Fig. 1. Acquisition scheme of multisweep C-arm scans where contrast is injected during one breathold over all sweeps. The projection data is gated retrospectively according to a reference cardiac phase.

it is highly variable in both temporal and spatial domains. The less complex respiratory motion can be regularized globally, and image artifacts should not significantly corrupt the motion estimate. The SNR enhancement introduced by Li et al. increases blurring when edges of different phases do not match perfectly, as is expected to be the case for artifact-prone cardiac reconstruction. Our weighting scheme addresses this problem. The improvement in image quality via the integration of data from different respiratory or cardiac phases to the desired phase is limited by the temporal resolution of each single reconstructed phase. We therefore chose an approach that aligns each acquired projection image to the desired phase. The projection data is gated into several subsets, where each subset provides data for a short-scan. Each motion corrected short-scan weights its contribution according to the expected or estimated confidence of the corrected data to the resulting reconstruction.

The paper is organized as follows. First, we briefly outline recent cardiac $\mathrm{C}$-arm CT acquisition protocols and introduce general notation for projection (sub)sets and common ECG-gating techniques. Then, our method for retrospective heart motion estimation and dynamic FDK-like reconstruction is presented. Two methods to enhance SNR by combining ECG-gated and motion corrected FDK-like reconstructions (provides sharp edges, but lower SNR) and dynamic FDK-like reconstructions utilizing the projection data from all sweeps (nongated) are introduced. The combined motion estimation and correction framework is evaluated using phantom data and an animal model. The results are summarized, and discussed with reference to other, previously published approaches.

\section{THEORETICAL FRAMEWORK}

\section{A. Retrospective ECG-Gating}

Before describing the algorithms in detail, we first introduce the basic notation and concepts. Projection images are denoted by bold face small and volumes by bold face capital letters. Sets are denoted by normal face capital letters. The concept of ECG-gated cardiac C-arm CT and the resulting distribution of projection images due to their relative cardiac phase is shown in Fig. 1 and summarized in Lauritsch et al. [2].

Let $\beta$ be the unique projection image $\boldsymbol{f}_{\beta}$ index of a multisweep scan, where $\beta=1, \ldots, N_{p}$ and $N_{p}$ is the total number of acquired projection images. $N_{s}$ is the number of sequential 
ECG-synchronized forward (fw) and backward (bw) sweeps. Each single sweep is a short scan. Thus, during one forward or backward sweep, $N_{p} / N_{s}$ projections of the calibrated view positions are acquired. The cardiac phase $t$ is defined by its position between R-peaks in the ECG signal and is measured in percent. Let $P$ be the set of all projection image indices $\beta$ and the cardinality is $|P|=N_{p}$. For a particular cardiac phase $t$ a gated subset is defined by $P^{t, w=0} \subseteq P$ and contains $N_{p} / N_{s}$ projection image indices. This gated subset is defined such that a single projection image index is provided for each single view position of a short scan. $P^{t, w=0}$ can be used for a short scan Feldkamp [1] reconstruction. The index $w$ denotes different gating window widths, as explained below. Since $N_{s}$ sweeps are performed, exactly $N_{s}$ projection images are measured for each single short scan view position. To reconstruct at a specific cardiac phase $t$ the projection image that lies closest to this reference cardiac phase is selected for each view position. Thus the subset $P^{t, w=0}$ contains the projection image indices of a nearest neighbor (NN) ECG-gating, where the temporal window width is smallest for $w=0$.

This is, however, not very dose efficient, since only $1 / N_{s}$ of the acquired data is used in $P^{t, w=0}$. We extend the gating and create further projection subsets $P^{t, w}$ for $w=1, \ldots, N_{s}-1$ that can be used for the reconstruction in combination with the weighting and motion compensation schemes introduced here. These sets are indexed by $w$ (gating windows)

$$
P^{t, w} \subseteq P \backslash\left\{P^{t, w=0}, \ldots, P^{t, w-1}\right\}
$$

and $P^{t, w_{i}} \cap P^{t, w_{j}}=\emptyset, \forall i \neq j$. Each subset $P^{t, w}$ for all $w=0, \ldots, N_{s}-1$ is gated using the same NN ECG-gating strategy, however only those remaining projection image indices as defined in (1) are considered. This gating approach groups projections into $N_{s}$ distinct subsets, each containing $1 / N_{s}$ of all $|P|$ acquired projections. As $w$ increases, the cardiac phases of the projections contained in a subset are farther from the reference cardiac phase $t$.

The cardiac phase function $\tau$ provides the observed cardiac phase of each projection image $\boldsymbol{f}_{\beta}$. The cardiac phase function operates on $\beta$ and maps each $\beta$ to its corresponding cardiac phase

$$
\tau: \beta \mapsto t
$$

Note that $\tau(\beta)$ is not necessarily equal to $t$.

We define the effective cardiac phase $(\mathrm{ECP}) \tau_{E}(t, w) \in$ $[0,100], \forall t \in[0,100], s=0, \ldots, N_{s}$, for a selected subset of projection images $P^{t, w}$ of cardiac phase $t$

$$
\tau_{E}\left(P^{t, w}\right)=\frac{1}{\left|P^{t, w}\right|} \sum_{\beta \in P^{t, w}} \tau(\beta) .
$$

The cardiac phase variance (CPV) of an ECG-gated subset $P^{t, w}$ is defined by

$$
\sigma_{C P}^{2}\left(P^{t, w}\right)=\frac{1}{\left|P^{t, w}\right|} \sum_{\beta \in P^{t, w}}(t-\tau(\beta))^{2} .
$$

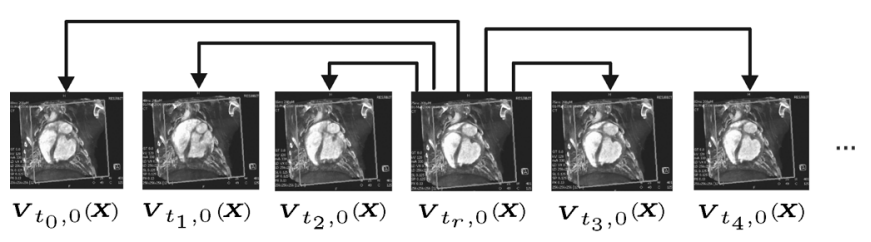

Fig. 2. Pairwise 3-D-3-D nonrigid registration of the initial reconstructions.

The gating method results in an increasing cardiac phase variance as $w$ increases, $\sigma_{C P}^{2}\left(P^{t, w=0}\right) \leq \sigma_{C P}^{2}\left(P^{t, w=1}\right), \ldots, \leq$ $\sigma_{C P}^{2}\left(P^{t, w=N_{s}-1}\right)$.

In retrospectively-gated cardiac $\mathrm{C}$-arm $\mathrm{CT}$, image quality depends on the motion of the heart and on the temporal resolution of the projection data contributing to a reconstruction. At acquisition, a cardiac phase $t$ such as end diastole is selected for which the heart motion is assumed to be negligible [2]. The timing of the scan (i.e., acquisition of backward and forward sweeps) is then triggered so that the variance $\sigma_{C P}^{2}\left(P^{t, w=0}\right)$ at the selected phase is minimized. The resulting temporal resolution of all reconstructions depends on the number $N_{s}$ of performed C-arm sweeps and is approximately $1 / N_{s}$ of the average $\mathrm{R}-\mathrm{R}$ interval of the entire imaging time. We use the term cardiac phase variance instead of temporal resolution to express the resulting temporal window width after retrospective gating. However, depending on $t$ and the heart motion, motion related blurring in an FDK reconstruction of $P^{t, w=0}$ at the selected phase and at other phases can still be observed. We first use an estimate of the subject-specific heart motion to reduce motion artifacts and increase edge sharpness of the reference cardiac volume. We then add projection data from other subsets $P^{t, w}$ for $w>0$ to this motion corrected reconstruction using novel weighting schemes that are described later in detail.

\section{B. Retrospective Motion Estimation}

If we neglect the implications of interpolation and tissue density variation during contraction, we can represent the temporal information of the volume data by either a sequence of volumes or by a single volume and a sequence of displacement vector fields. In our scenario the input to the registration method is volume data and the output is a coordinate transform from one volume to the other as shown in Fig. 2. For computation of the displacement vector fields a temporal sequence of initial reconstructions is required.

1) Initial Reconstructions: The reconstruction task is the computation of object densities for all grid points of a particular volume from projections. All volumes herein are cubes of size $N \times N \times N$ voxels with 3 -D grid points $\boldsymbol{X}=\left\{\boldsymbol{x}_{j}=\left(x_{j}, y_{j}, z_{j}\right)^{T} ; j=1,2, \ldots, N^{3}\right\}$. The object density for a particular voxel $\boldsymbol{x}_{j} \in \boldsymbol{X}$ of an ECG-gated FDK reconstruction (EG-FDK) using the projection set $P^{t, w}$ is denoted by $V_{t, w}\left(x_{j}\right) \in$, and $V_{t, w}(\boldsymbol{X}) \in^{N \times N \times N}$ is the whole volume of intensity values. To compute the subject-specific heart motion, a cardiac phase series $t_{i}$ of $N_{b}$ volumes (see Fig. 2) $\boldsymbol{V}_{t_{i}, 0}(\boldsymbol{X})$ for $i=0, \ldots, N_{b}-1$ is initially reconstructed using EG-FDK. 
For these initial reconstructions only the gating window $w=$ 0 is used.

2) Nonrigid Registration: The heart motion is estimated voxel-wise and relative to a preselected reference cardiac phase $t_{r}$ (RCP) that is by definition one of the $N_{b}$ phases $t_{i}$. A dense displacement vector field $\boldsymbol{U}_{t_{i}}=\left\{\boldsymbol{u}_{j}^{t_{i}}=\left(u_{j}^{x}, u_{j}^{y}, u_{j}^{z}\right)^{T} ; j=1,2, \ldots, N^{3}\right\}$ maps the coordinates of the reference volume $\boldsymbol{V}_{t_{r}, 0}(\boldsymbol{X})$ to any target volume $\boldsymbol{V}_{t_{i}, 0}(\boldsymbol{X})$. In discrete space the 3 -D displacement vector $\boldsymbol{u}_{j}^{t_{i}}$ maps a discrete grid point $\boldsymbol{x}_{j}$ of the reference volume to a 3-D point $\boldsymbol{y}_{j}=\boldsymbol{x}_{j}+\boldsymbol{u}_{j}^{t_{i}}$ in the target volume. The 3-D point in the target volume is not necessarily a grid point, and 3-D interpolation is required to access the corresponding function value of the reference grid point $\boldsymbol{x}$ in the deformed target volume. The displacement vector field is computed by performing 3-D-3-D nonrigid registration between all volume pairs $\left(\boldsymbol{V}_{\boldsymbol{t}_{i}, 0}(\boldsymbol{X}), \boldsymbol{V}_{\boldsymbol{t}_{r}, 0}(\boldsymbol{X})\right)$, for all $i=1, \ldots, N_{b}-1$ as shown in Fig. 2. The volume of the reference cardiac phase $t_{r}$ is mapped to all the other (deformed) volumes, providing the relative heart motion from $t_{r}$ to $t_{i}$ for all $i$.

The registration problem is defined as a variational problem with the energy functional

$$
\mathcal{I}\left(\boldsymbol{U}_{t_{i}}\right)=\mathcal{J}\left(\boldsymbol{U}_{t_{i}}\right)+\alpha \mathcal{R}\left(\tilde{U}_{t_{i}}\right)
$$

consisting of a dissimilarity measure

$$
\mathcal{J}\left(\boldsymbol{U}_{\boldsymbol{t}_{i}}\right)=\sum_{j}\left(\boldsymbol{V}_{\boldsymbol{t}_{i}, 0}\left(\boldsymbol{x}_{j}+\boldsymbol{u}_{j}^{t_{i}}\right)-\boldsymbol{V}_{\boldsymbol{t}_{r}, 0}\left(\boldsymbol{x}_{j}\right)\right)^{2}
$$

and the irregularity of the dense deformation field

$$
\mathcal{R}\left(\boldsymbol{U}_{t_{i}}\right)=\sum_{j}\left(\Delta \boldsymbol{u}_{j}^{t_{i}}\right)^{2} .
$$

To minimize the energy functional we compute its first variation and use a time-marching method to find the $\boldsymbol{U}_{t_{i}}$ that minimizes $\mathcal{I}$. A fast direct cosine transform technique [13] can be used with a multilevel approach to increase computation speed and handle larger spatial deformations. The deformation is regularized by $\mathcal{R}$ with weight $\alpha$. Each pair can be computed in parallel since the minimization can be done for each phase $t_{i}$ independently. The advantage of parallel computation comes at the expense of temporal smoothness, since any additional temporal regularization (i.e., beyond $\mathcal{R}$ ) is computationally more expensive. More details about parameter settings are given in Section III-A1.

3) Interpolation of the Deformation Field: The next step is to concatenate the discrete time series of MVFs $\boldsymbol{U}_{t_{i}}$ so that the MVFs are cyclic and continuous over the cardiac phase. For later motion correction the $U_{t}$ for $t=\tau(\beta), \forall \beta \in P^{t, w}$ are computed using interpolation. The displaced locations $\boldsymbol{x}_{j}+\boldsymbol{u}_{j}^{t_{i}}$ of each voxel define the discrete temporal samples $t_{i}$ used as knot points for the interpolation function. The $\boldsymbol{U}_{t}$ are then the interpolated MVFs for phase $t$. It is by definition cyclic such that $\boldsymbol{U}_{t=0}=\boldsymbol{U}_{t=100}$ and $\boldsymbol{U}_{t_{r}}=0$. Different interpolation kernels such as nearest neighbor, linear, cubic-spline or polynomial interpolation have been investigated in [19].

\section{Motion Compensated FDK (MC-FDK)}

We implement a dynamic filtered backprojection (FBP) algorithm using FDK [1], the work horse in C-arm CT, where the ge- ometry of cone-beam backprojection is adapted using the computed motion model. For a detailed description of cone-beam filtered backprojection we refer to the original work from Feldkamp et al. [1].

For a MC-FDK reconstruction of cardiac phase $t$ a set $P^{t, w}$ of projection images is used. Each projection image $f_{\beta}$ is filtered row wise [1]. The filtered image, $\tilde{\boldsymbol{f}}_{\beta}$, is then backprojected into 3-D, denoted by $\tilde{\boldsymbol{F}}_{\beta}(\boldsymbol{X}) \in^{N \times N \times N}$. The standard FDK algorithm then accumulates all $\tilde{\boldsymbol{F}}_{\beta}(\boldsymbol{X})$ into one 3 -D volume. To compensate for motion, each $\tilde{\boldsymbol{F}}_{\beta}(\boldsymbol{X})$ is spatially warped $\tilde{\boldsymbol{F}}_{\beta}\left(\boldsymbol{X}+\boldsymbol{U}_{\tau(\beta)}\right)$ according to the estimated dense deformation $\boldsymbol{U}_{t=\tau(\beta)}$ before this accumulation step. This motion compensation is equivalent to backprojection along a curved ray, as though the forward projection had been created by integrating the tissue densities along the curved projection ray as defined by the MVF. The interpolation along the curved rays can be implemented in the projection space during backprojection or in a second interpolation step in 3-D after backprojecting straight rays. Cosine and Parker [14] weights are not adapted to the dynamic FBP.

1) Warping in Projection Space ( $M C-F D K-P)$ : Numerically, the better choice is to directly sample the 2-D filtered projection image according to the warped positions $\boldsymbol{X}+\boldsymbol{U}_{t}$. Using a voxel-driven backprojection we first transform the 3-D volume grid from $\boldsymbol{X}$ to $\boldsymbol{X}+\boldsymbol{U}_{t}$ and then use cone-beam projection geometry to compute the 2-D sampling position of the warped 3 -D grid position. The projected grid position does not necessarily intersect the 2-D detector at lattice points. Thus efficient 2-D linear interpolation is required. The projection of arbitrary warped grid points is time consuming.

2) Warping in Backprojection Space (MC-FDK-B): To take advantage of fast backprojectors that expect equidistant grid positions $\boldsymbol{X}$, warping can also be done after backprojection. The filtered projection $\tilde{\boldsymbol{f}}_{\beta}$ is backprojected onto $\boldsymbol{X}$ and then $\tilde{\boldsymbol{F}}_{\beta}(\boldsymbol{X})$ is spatially warped according to the MVF such that we get $\tilde{\boldsymbol{F}}_{\beta}\left(\boldsymbol{X}+\boldsymbol{U}_{\tau(\beta)}\right)$. However, since a 2-D interpolation of the filtered projections is followed by a trilinear interpolation during the spatial warping of $\tilde{\boldsymbol{F}}_{\beta}\left(\boldsymbol{X}+\boldsymbol{U}_{\tau(\beta)}\right)$, this approach accumulates interpolation errors as compared with warping applied directly to each projection (i.e., in projection space).

We implemented sampling in the backprojection space, and provide results of MC-FDK using only this sampling method. Using an ECG-gated set $P^{t, w}$ to reconstruct phase $t$ of any gating window width $w$, the MC-FDK algorithm can be illustrated as follows (see Fig. 3).

1) During back-projection, we first apply standard FDK-based filtering and voxel-driven back-projection separately for each $\beta \in P^{t, w}$.

2) Each filtered and 3-D back-projected image $\tilde{\boldsymbol{F}}_{\beta}(\boldsymbol{X})$ is spatially warped by regridding the filtered back-projection (FBP) according to $U_{\tau(\beta)}$.

3) All $\left|P^{t, w}\right|$ spatially warped filtered backprojections are accumulated according to

$$
\hat{\boldsymbol{V}}_{t, w}(\boldsymbol{X})=\sum_{\beta \in P^{t, w}} \tilde{\boldsymbol{F}}_{\beta}\left(\boldsymbol{X}+\boldsymbol{U}_{\tau(\beta)}\right)
$$

to create the final 3-D motion corrected volume $\hat{\boldsymbol{V}}_{t, w}(\boldsymbol{X}) \in^{N \times N \times N}$.

Note that assumptions underlying FBP, such as the Fourierslice theorem and uniform sampling density, do not hold for 


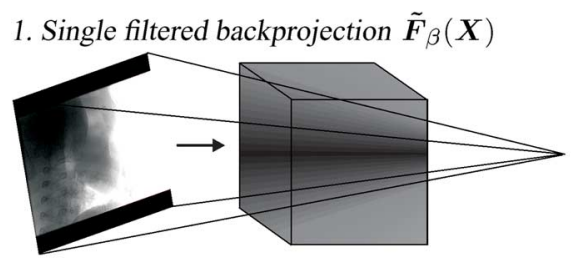

2. Spatial warping $\tilde{\boldsymbol{F}}_{\beta}\left(\boldsymbol{X}+\boldsymbol{U}_{\tau(\beta)}\right)$

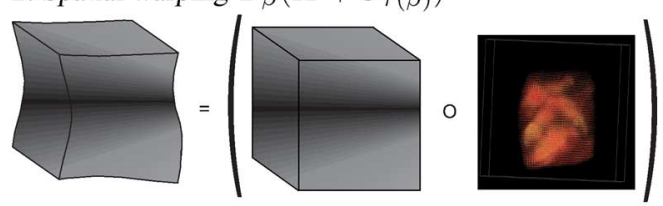

3. Accumulation of motion corrected FBPs eqn. (8)

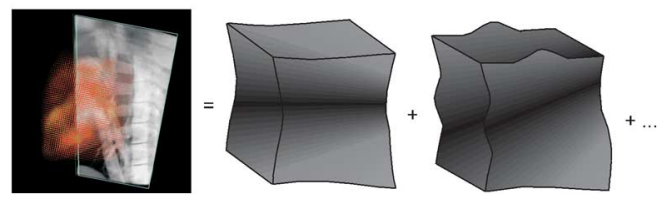

Fig. 3. Principal steps of a dynamic FDK-like algorithm (MC-FDK). The left image of the third step shows the reconstructed volume, overlayed with a MVF. The right hand side shows the accumulation of the warped filtered back-projections.

curved rays in general, and that rebinning of data into a parallel ray geometry is not possible if the data was acquired during arbitrary motion. Thus even when the MVF is known perfectly, the motion corrected reconstruction may have artifacts resulting from nonuniform sampling of the volume data and from nonideal filtering of the projection data. We assume here that the heart motion is sufficiently small and we do not consider any sampling density or filter compensation; the impact of this assumption is evaluated below using a numerical simulation study.

\section{Application: SNR Enhanced Reconstruction}

The introduced framework of motion estimation and correction provides a tool to utilize all acquired projection images from a multisweep scan in combination with motion correction. Use of more than $\left|P^{t, w}\right|$ images may provide increased SNR, while the MVF correction preserves the sharpness of the image's anatomical structure. In this application we investigate the motion estimation and correction framework for contrast enhanced ventricles of the heart.

We derive and compare three weighting schemes that combine motion corrected reconstructions using the ECG-gated sets $P^{t, w}$ into a single volume at the reference cardiac phase $t_{r}$ such that all $|P|$ projections contribute. The ECG-gated subsets $P^{t_{r}, w}$ provide data for $N_{s}$ motion corrected reconstructions $\hat{\boldsymbol{V}}_{t, w}(\boldsymbol{X})$ where each reconstruction $w=0, \ldots, N_{s}-1$ is generated using the gated subset $P^{t_{r}, w}$ targeting $t_{r}$.

Our weighting schemes consider the following three observations.

- Estimation of subject-specific heart motion is imperfect and accuracy depends on the image quality (edge sharpness) of the initial reconstructions.

- Deformation between a volume in systole and a volume in diastole is larger than the deformation seen between two volumes at different time points in diastole. The MC-FDK becomes less accurate as the nonrigid spatial deformation to be corrected becomes larger.

- The reconstruction using $P^{t, w=0}$ provides the sharpest edges and the best image quality but is not optimal in SNR.

1) Averaging Scheme (SNRO): One method to combine all $|P|$ projections, SNR0, is to take a voxel-by-voxel average of the reconstructed intensity of all resulting $w=0, \ldots, N_{s}-1$ gating windows. The resulting SNR enhanced reconstruction is $\hat{V}_{t_{r}}^{E} \in^{N \times N \times N}$ where

$$
\hat{\boldsymbol{V}}_{\boldsymbol{t}_{r}}^{E}\left(\boldsymbol{x}_{j}\right)=\frac{1}{M_{j}} \sum_{w=0}^{N_{s}-1} C_{j}^{t_{r}, w} \hat{\boldsymbol{V}}_{t_{r}, w}\left(\boldsymbol{x}_{j}\right)
$$

First each voxel $x_{j}$ is summed with weighting $C_{j}^{t_{r}, w}$ which for simple averaging is set to $C_{j}^{t_{r}, w}=1$. The sum is then normalized by $M_{j}=N_{s}$. This method trades off spatial resolution for SNR since the motion compensated reconstructions using the gating windows $w>0$ provide blurred edges compared to $w=0$. Thus we extend the weighting method and introduce two adapted schemes.

2) Cardiac Phase Variance Scheme (SNR1): Given the assumptions regarding window width, motion and resolution, we propose a weighting that is adapted to the cardiac phase variance for each single motion corrected reconstruction $\hat{\boldsymbol{V}}_{t_{r}, w}$, SNR1. The contribution is calculated using a Gaussian function with standard deviation $\sigma_{a}$, defined by

$$
\begin{aligned}
d_{\mathrm{SNR} 1}^{t_{r}, w} & =\sigma_{C P}^{2}\left(P^{t_{r}, w=0}\right)-\sigma_{C P}^{2}\left(P^{t_{r}, w}\right) \\
C_{j}^{t_{r}, w} & :=\frac{1}{\sqrt{2 \pi}} \exp \left(-\frac{\left(d_{\mathrm{SNR} 1}^{t_{r}, w}\right)^{2}}{2 \sigma_{a}^{2}}\right) .
\end{aligned}
$$

The normalization function is then given by

$$
M_{j}:=\sum_{w=0}^{N_{s}-1} C_{j}^{t_{r}, w} .
$$

According to (10), the MC-FDK reconstruction using the set $P^{t_{r}, w=0}$ contributes most to the resulting image $\hat{V}_{t_{r}}^{E}$ as defined in (9). With increasing cardiac phase variance, the MC-FDK reconstructions contribute less in order to preserve edge sharpness. Each voxel is given the same weight for a specific $w$.

3) Intensity Scheme (SNR2): The second extended weighting method, SNR2, assumes that the motion corrected reconstruction $\hat{\boldsymbol{V}}_{t_{r}, w=0}(\boldsymbol{X})$ provides the sharpest edges and provides a good reference volume. The contribution function is based on a Gaussian function with standard deviation $\sigma_{b}$. The intensity similarity between the reference $w=0$ and all others $w>0$ define the contribution weight with

$$
\begin{aligned}
& d_{\mathrm{SNR} 2}^{t_{r}, w, j}=\hat{V}_{t_{r}, w=0}\left(\boldsymbol{x}_{j}\right)-\hat{\boldsymbol{V}}_{t_{r}, w}\left(\boldsymbol{x}_{j}\right) \\
& C_{j}^{t_{r}, w}:=\frac{1}{\sqrt{2 \pi}} \exp \left(-\frac{\left(d_{\mathrm{SNR} 2}^{t_{r}, w, j}\right)^{2}}{2 \sigma_{b}^{2}}\right) .
\end{aligned}
$$

The normalization function $M_{j}$ of (11) is used, but with $C_{j}^{t_{r}, w}$ as defined in (12). The motion correction depends on the subject-specific MVF estimate and the accuracy of this estimate is 
TABLE I

Number of PROJECTION IMAGES CONTRIBUTING TO EACH RECONSTRUCTION METHOD

\begin{tabular}{ccccccc}
\hline EG-FDK & AV-FDK & MC-FDK & SNR0 & SNR1 & SNR2 & AL-EG-FDK \\
$\left|P_{t, w}\right|$ & $N_{p}$ & $\left|P_{t, w}\right|$ & $N_{p}$ & $\leq N_{p}$ & $\leq N_{p}$ & $N_{p}$ \\
\hline
\end{tabular}

uncertain in a clinical environment. This uncertainty is therefore implicitly included in the success of motion blurring reduction. If the intensity deviation of a MC-FDK reconstruction is high according to (12), the reconstructed intensity value will contribute less to the resulting reconstruction. The more the motion corrected reconstructions reflect the $\hat{V}_{t_{r}, w=0}(\boldsymbol{X})$ reference the higher the contribution will be.

4) Algorithmic Summary of SNR Enhanced Reconstruction: The algorithmic pipeline of this reconstruction system is summarized in algorithm 1 .

Algorithm 1 SNR Enhanced Reconstruction

1) Reconstruction of $i=0, \ldots, N_{b}-1$ volumes $\boldsymbol{V}_{t_{i}}(\boldsymbol{X})$ using ECG-gated FDK.

2) Set $t_{r}$.

3) Computation of $\boldsymbol{U}_{t_{i}}$ and $i=0, \ldots, N_{b}-1$ using (5).

4) Temporal interpolation of $\boldsymbol{U}_{\tau(\beta)}, \forall \beta \in P$.

5) Reconstruct $\hat{\boldsymbol{V}}_{\boldsymbol{t}_{r}, \boldsymbol{w}}(\boldsymbol{x})$ for $w=0, \ldots, N_{s}-1 \mathrm{using}$ MC-FDK.

6) Compute $C_{j}^{t_{r}, w}$ and $M_{j}$ depending on weighting scheme SNR0 $\left(C_{j}^{t_{r}, w}=1\right)$, SNR1 (10) or SNR2 (12).

7) Compute $\hat{\boldsymbol{V}}_{\boldsymbol{t}_{r}}^{E}(\boldsymbol{X})$ using (9).

5) Algorithmic Variations: We compare SNR0, SNR1, and SNR2 to a nonmotion compensated, but SNR enhanced reconstruction, AL-EG-FDK. $N_{b}$ nonrigidly aligned initial reconstructions are averaged

$$
\boldsymbol{V}_{t_{r}}^{\mathrm{AL}-\mathrm{EG}}(\boldsymbol{X})=\frac{1}{N_{b}} \sum_{i=0}^{N_{b}-1} \boldsymbol{V}_{t_{i}, w=0}\left(\boldsymbol{X}+\boldsymbol{U}_{t_{i}}\right) .
$$

We also compute a nongated FDK reconstruction, AV-FDK, using all acquired projection images $|P|$

$$
\boldsymbol{V}_{\boldsymbol{t}_{r}}^{\mathrm{AV}}(\boldsymbol{X})=\frac{1}{|P|} \sum_{\beta \in P} \tilde{\boldsymbol{F}}_{\beta}(\boldsymbol{X}) .
$$

A complete list of all algorithmic variation is presented in Table VII. We investigate these algorithmic variations in the result section and demonstrate their performance. The number of images contributing to the final reconstruction for each method is summarized in Table I.

\section{EXPERIMENTAL METHODS}

To evaluate the image quality of the introduced methods listed in Table VII, we measure and compare the SNR and the edge response function [18] to state-of-the-art reconstructions methods. The evaluation of the algorithms is carried out using a moving plastic phantom and several animal models. For the phantom, we also compare our approaches with a standard Feldkamp reconstruction of the static object, the ground truth (GT-FDK).

\section{A. Motion Estimation}

The cardiac phases of the initial reconstructions that are used to compute the MVF are selected manually. The selection process considers prior knowledge of the expected heart motion and the sharpness of the reconstructed volumes.

1) Registration Method Parametrization: For the nonrigid registration we use spatial regularization only. Empirical studies showed that temporal regularization does not significantly improve image quality. For both phantom and in vivo studies we applied a multilevel scheme with four levels ([volume size, iterations], [256 $\left.\left.36^{3}, 5\right],\left[128^{3}, 20\right],\left[64^{3}, 90\right],\left[32^{3}, 140\right]\right)$, and for the spatial curvature regularization $\alpha=0.2$.

2) Temporal Interpolation: In previous studies, we determined empirically that cubic spline and linear interpolation outperform nearest neighbor and polynomials, and so here all temporal interpolation for the computation of $U_{t}$ was restricted to cubic-spline.

\section{B. Simulation, In Vitro, and Animal Study}

1) Simulated Phantom: To evaluate the sampling issue that is raised in the MC-FDK algorithm, a simulation study in 2-D is performed. A modified Shepp-Logan phantom (see Fig. 4) is virtually scanned (parameters summarized in Table II), while the phantom undergoes nonrigid and sinusoidal warping during the scan. Dense horizontally oriented bars were added to the phantom to emphasize motion related blurring artifacts. We assume that the ideal MVF is known and compare, using the ideal MVFs, the MC-FDK method where the interpolation (warping)

- takes place in the projection-space (MC-FDK-P);

- is performed after the backprojection in the 3-D backprojection-space (MC-FDK-B).

These results are compared to AV-FDK and AL-EG-FDK.

2) In Vitro Phantom: The in vitro object consists of a plastic cube moving along a sinusoidal path in a water bath. The phantom was scanned using an artificial ECG signal that was connected to the $\mathrm{C}$-arm system during sinusoidal motion of the cube along the axis of rotation of the $\mathrm{C}$-arm. The artificial RR-peak of the phantom $(0 \%)$ occurred at a position of -7 $\mathrm{mm}$. Maximum velocity occurred at $25 \%$ and $75 \%$ and the maximum amplitude of $-7 \mathrm{~mm}$ and $7 \mathrm{~mm}$ occurred at $0 \%$ and $50 \%$. The protocol is presented in Table III. We chose to investigate a phase for which phantom velocity was close to the maximum (i.e., $\approx 84 \%$ ), in order to fully challenge our motion correction algorithms. A more typical choice would consider a phase with minimum velocity for the reference.

3) Animal Study: The research protocol was approved by the Institutional Animal Care and Use Committee at Stanford University. An adult swine $(\approx 40 \mathrm{~kg})$ was first anesthetized using intramuscular injection of ketamine; the animal was then intubated and given a mixture of oxygen and isoflurane. An 8 French introducer sheath was placed in the femoral artery and femoral vein for hemodynamic monitoring and administration of medications and contrast material. Both animal and plastic cube were 


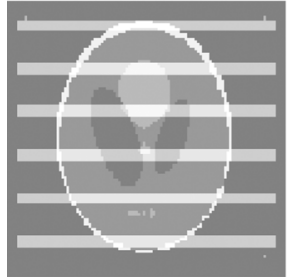

(a)

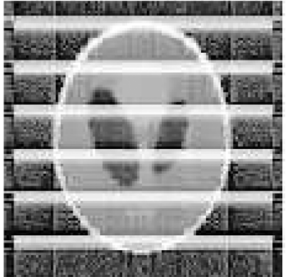

(b)

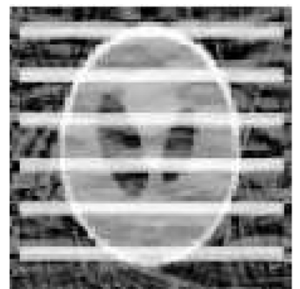

(c)

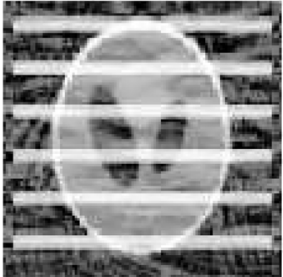

(d)

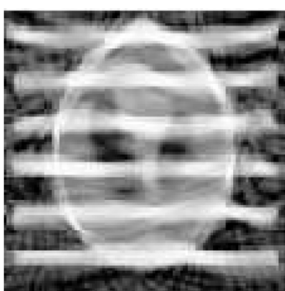

(e)

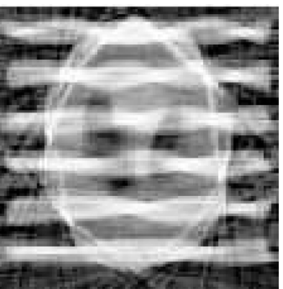

(f)

Fig. 4. Simulation study using an ideal MVF. The figure shows a modified Shepp-Logan phantom using the reconstruction method: (a) original phantom, (b) filtered-backprojection of nonmoving object, (c) MC-FDK-B, (d) MC-FDK-P, (e) AL-EG-FDK, and (f) a filtered-backprojection without motion correction (AVFDK).

TABLE II

MODIFIED SHEPP-LOGAN PHANTOM PROTOCOL

\begin{tabular}{ll}
\hline$N_{s}$ & 4 \\
$N_{p}$ & 640 \\
$\left|P_{t, w}\right|$ & 160 \\
$\tau_{E}\left(P^{t, w=0}\right)$ & $25,50,75,100$ \\
$\sigma_{C P}\left(P^{t, w=0}\right)$ & $0.05,0.2,0.1,0.07$ \\
Maximum motion amplitude & 4.5 pixel (unit length) \\
Size $N$ & $128 \times 128$ pixel (slice) \\
\hline
\end{tabular}

TABLE III

IN VITRO PHANTOM PROTOCOL

\begin{tabular}{ll}
\hline$N_{s}$ & 4, ECG-synchronized (each $4 s$ ) \\
$N_{p}$ & 764 \\
$\left|P_{t, w}\right|$ & 191 \\
$\tau_{E}\left(P^{t, w=0}\right)$ & $12,35,56,73,84,91$ \\
$\sigma_{C P}^{2}\left(P^{t_{r}, w}\right)$ for $w=0,1,2,3$ & $0.0058,0.0354,0.1005,0.1869$ \\
Motion amplitude & $+/-7 \mathrm{~mm}^{3}$ \\
Voxel size & $0.76 \mathrm{~mm}^{3}$ \\
Cycles per second & 1 \\
$t_{r}$ & 84 \\
$N_{b}$ & 6 \\
Nyquist & $6.49 \mathrm{lp} / \mathrm{cm}$ \\
\hline
\end{tabular}

TABLE IV

ANIMAL STUDY PROTOCOL

\begin{tabular}{ll}
\hline$N_{s}$ & 6$, ECG-synchronized (each $4 s)$ \\
$N_{p}$ & 1146 \\
$\left|P_{t, w}\right|$ & 191 \\
$\tau_{E}\left(P^{t, w=0}\right)$ & $4,29,43,78,94$ \\
$\sigma_{C P}\left(P^{t_{r}, w}\right)$ & $0.0054,0.0240,0.0507,0.0787,0.1260,0.1924$ \\
Voxel size & $0.76 \mathrm{~mm}^{3}$ \\
$t_{r}$ & 94 \\
$N_{b}$ & 5 \\
Nyquist & $6.49 l p / \mathrm{cm}$ \\
\hline
\end{tabular}

scanned using an AXIOMArtis dTA C-arm system. During the scan $175 \mathrm{ml}$ of Omnapaque was injected into the vena cava (injection rate $3.5 \mathrm{ml} / \mathrm{s}$ ). The protocol is summarized in Table IV.

\section{Evaluation of Image Quality}

To measure the image quality we compute the edge response function and the SNR.

1) Edge Response-Function: The edge response function is computed by averaging together shifted edge profiles; shifting ensures that the center of each profile corresponds to the center of the edge. For the in vitro phantom, 30 profiles along a straight line perpendicular to the direction of sinusoidal motion were first shifted to align the center and then averaged together. For the animal study, 50 edges were measured perpendicular to the boundary between the interventricular septum and the papillary muscle. To detect and align the edges we use a structure tensor [16] where the edge candidates are those for which the ratio of the eigenvalues from the structure tensor matrix is larger than a manually selected threshold. This technique automatically detects the orientation and center of edges. We sample using the nearest neighbors along the line that is perpendicular to the edge and average several centered profiles of the ventricles' edge. For the simulated phantom, the straight edge of a dense horizontal bar is known and used for evaluation. The averaged edge is convolved with a step-size function and then the Fourier transform of the resulting first derivative of the edge is taken. The edge response function is then normalized to the zero frequency of the Fourier transformed signal.

2) Signal-To-Noise Ratio: Using the volume rendering software InSpace [17] a manually selected volume of interest (VOI) is placed inside a contrast-filled ventricle or in the in vitro phantom. We compute the SNR as the ratio of the mean voxel intensity value to the standard deviation inside the VOI region.

\section{RESULTS}

We first consider numerical simulations of the sampling issue during the spatial warping of the filtered back-projections as described in the MC-FDK algorithm II-C. Then we demonstrate the performance of the introduced motion correction method on the in vitro phantom and on an animal subject.

\section{A. Simulation Study}

The reconstructed images of the simulated phantom are shown in Fig. 4. The two right images show the result of (e) AL-EG-FDK and (f) AV-FDK, with strong motion blurring. The ground truth phantom is shown in (a) and the 2-D FBP of the nonmoving phantom is shown in (b). An important question is the interpolation of warped rays during backprojection as described in Section II-C. In this study we assume that the ideal MVF is known and compare, using the ideal MVFs, the MC-FDK method where the interpolation (warping)

- takes place in the projection-space (MC-FDK-P);

- is performed after the backprojection in the 3-D backprojection-space (MC-FDK-B).

Visually there is no significant difference between the motion corrected reconstructions using (c) MC-FDK-P and (d) MC-FDK-B. The corresponding edge response functions are 


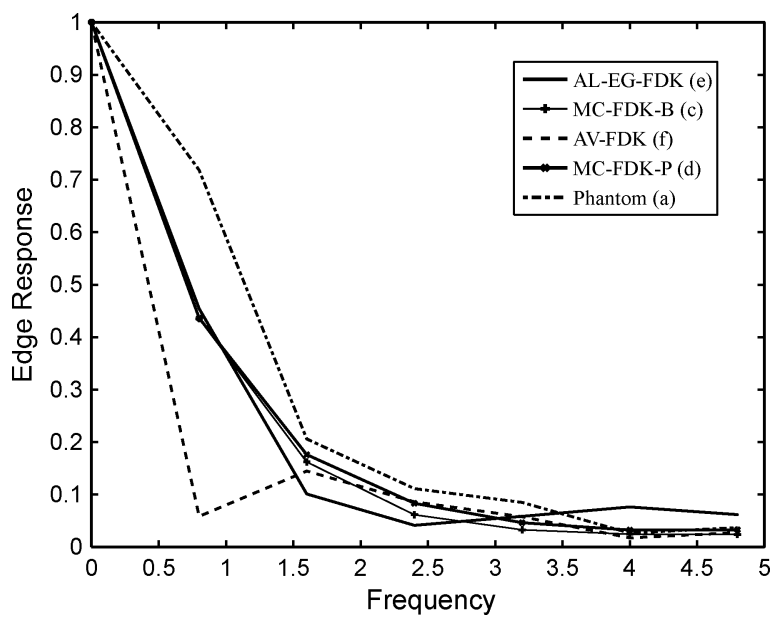

Fig. 5. Edge response of simulated phantom using the reconstruction methods AV-FDK, MC-FDK-P, MC-FDK-B and AL-EG-FDK. The labels $(a, b, \ldots)$ denote the corresponding images in Fig. 4.

presented in Fig. 5. The edge response using the (c) MC-FDK-P method is slightly improved compared to the (d) MC-FDK-B method. However, the simulation shows that the gain in spatial resolution is only marginal compared to the computational cost of the more complex backprojection of the warped grid positions $\boldsymbol{X}+\boldsymbol{U}_{t_{i}}$. The result using AL-EG-FDK (e) shows a slight improvement compared to (f) AV-FDK due to the ECG-gated reconstruction of the four cardiac phases, before aligning them according to the ideal MVF. Averaging several ECG-gated reconstructions (e) that have been warped to one single cardiac phase still produces strong motion related blurring artifacts, even when the ideal MVF is applied. For the experiments that follow, interpolation according to MC-FDK-B was applied.

\section{B. In Vitro Phantom Study}

The edge profile of all seven reconstruction methods is shown in Fig. 7. The FDK reconstruction of the nonmoving phantom (GT-FDK) provides a reference against which all other methods can be compared. For the GT-FDK, EG-FDK, and MC-FDK, 191 projections were used for the reconstruction. For AL-EG-FDK, SNR0, SNR1, SNR2, and AV-FDK all 764 projections were considered during reconstruction. However, depending on the weighting schemes of SNR1 and SNR2, not all 764 projection images provided the same contribution to the final reconstruction. As shown in Fig. 7, the edge of the EG-FDK is strongly blurred and increases approximately linearly between the minimum and maximum of the motion amplitude. The sharpest edge, with a width of approximately $3 \mathrm{~mm}$ and a height of 300 intensity units, is provided by the GT-FDK reconstruction of the nonmoving phantom. Motion artifacts having an intensity offset of about 50 units can be observed in the methods EG-FDK and AL-EG-FDK, where the edge is also strongly blurred. All motion corrected reconstructions MC-FDK, SNR0, SNR1 and SNR2 provide increased edge amplitude as compared to the standard EG-FDK reconstruction.

The resulting edge response functions are shown in Fig. 8. The impact of the standard deviations $\sigma_{a}$ and $\sigma_{b}$ of SNR1 and

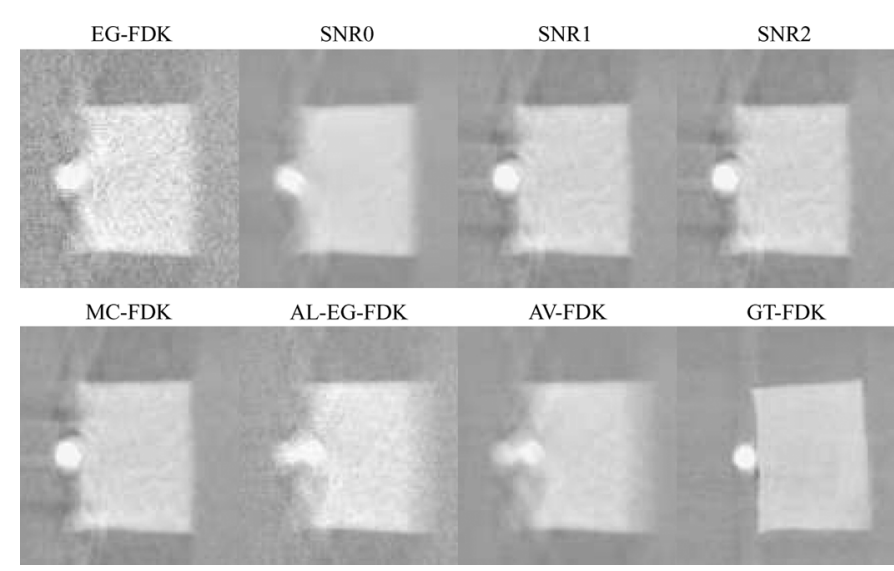

Fig. 6. Multiplanar rendered reconstructions of the in vitro phantom. The figure shows all reconstruction methods compared to the nonmoving object reconstruction GT-FDK. The sinusoidal motion of the phantom was from left to right according to the shown MPRs.

SNR2 are shown in Fig. 9. The resulting 30\% edge response versus the SNR for the range $\sigma_{a}=0.005,0.01, \ldots, 0.25$ and $\sigma_{b}=0.5,4.5, \ldots, 196.5$ is shown. The SNR and edge response for both SNR1 and SNR2 methods correspond to the measurements provided from the MC-FDK method. For small standard deviations such as $\sigma_{a}=0.005$ and $\sigma_{b}=0.5$, almost the same projections that are used for the MC-FDK reconstruction contribute to the SNR1 and SNR2 result. With increasing $\sigma_{a}$ and $\sigma_{b}$ more projections outside the targeted reconstruction window contribute to the final reconstruction. Thus, the SNR of the SNR1 and SNR2 methods increases up to the SNR0 measurements, where all acquired projection images contribute with the same weight to the final reconstruction. Here we observe a trade-off between increasing SNR and spatial resolution. This reflects the approximate motion estimation and correction where the spatial resolution drops as more motion corrected filtered backprojections contribute to the final reconstruction. The SNR2 provides a larger SNR compared to SNR1 for the same spatial resolution. The highest SNR is provided by AV-FDK, although the spatial resolution decreases to $0.675 \mathrm{lp} / \mathrm{cm}$. The SNR0 provides a comparable spatial resolution to EG-FDK, while the SNR of SNR0 is significantly increased. The AL-EG-FDK method provides an increased SNR compared to EG-FDK $(0.725 \mathrm{lp} / \mathrm{cm})$, however the edges become strongly blurred and the spatial resolution drops below $0.675 \mathrm{lp} / \mathrm{cm}$.

A multiplanar reconstruction of all methods is shown in Fig. 6 (window width $=587$, center $=1012$ ). The reference reconstruction GT-FDK of the nonmoving object is shown in the bottom right image. The EG-FDK is shown in the top left image. The edges perpendicular to the object's motion are significantly more blurred in the EG-FDK compared to all motion corrected reconstructions (MC-FDK, SNR0, SNR1, and SNR2). For SNR1 and SNR2, the standard deviations $\sigma_{a}=0.05$ and $\sigma_{b}=36.5$ have been used. We observed that the AL-EG-FDK method (13) does not improve edge sharpness although the same MVFs have been used to align the initial reconstructions to $t_{r}$. 


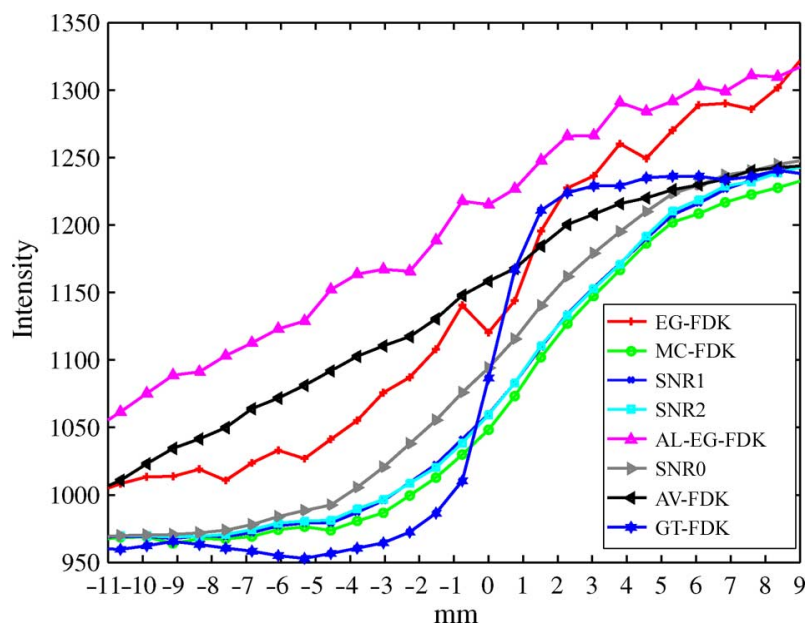

Fig. 7. Edge profiles of the in vitro phantom. The representative edges were computed via averaging 30 center aligned edge measurements. The edge is perpendicular to the sinusoidal motion direction. The centered positions do not represent any temporal specific edge locations. Standard deviation for the SNR1 and SNR2 method is $\sigma_{a}=0.05, \sigma_{b}=36.5$. The SNR1 profile is almost identical to SNR2.

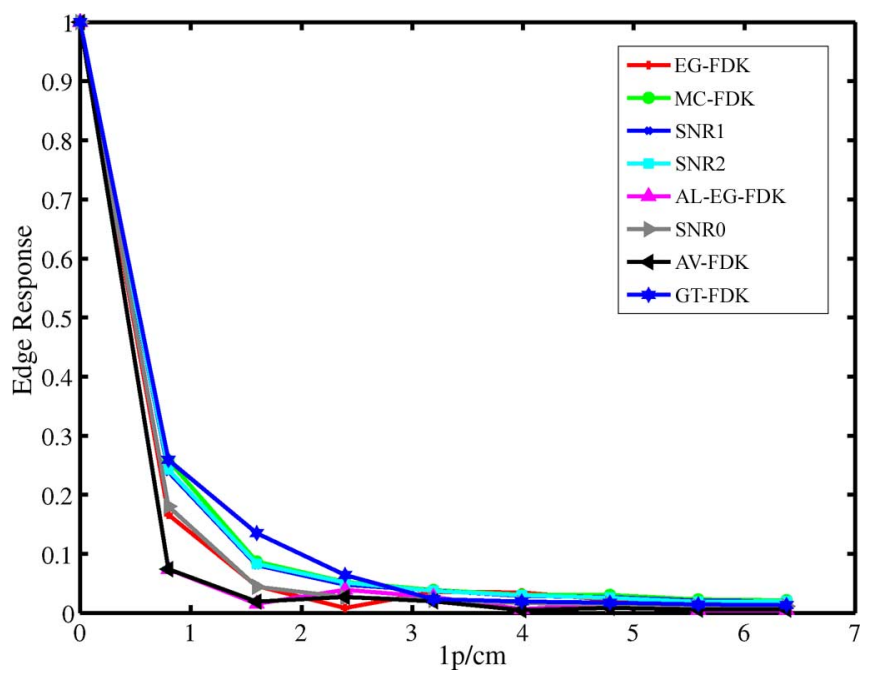

Fig. 8. In vitro phantom: Edge response of all reconstruction methods using $\sigma_{a}=0.05, \sigma_{b}=36.5$.

\section{Results in the Animal Model}

An intensity profile of all reconstruction methods measured across the ventricle is shown in Fig. 11. The edges of the AL-EG-FDK and AV-FDK method are strongly blurred. The left blurred edge is about $15 \mathrm{~mm}$ wide where the motion of the ventricle is large. The EG-FDK method provides a sharper time resolved edge, but inside homogeneous regions, the residual motion-related artifact contributes to a jagged profile. The motion corrected MC-FDK, SNR1, and SNR2 methods are more homogeneous with a comparable edge sharpness. The edges provided by the SNR0 method are slightly more blurred than EG-FDK.

The resulting edge response function measured around the papillary muscle is shown in Fig. 12. The MC-FDK method provides the best edge response followed by the SNR2 $\left(\sigma_{b}=28.5\right)$, EG-FDK, SNR1 $\left(\sigma_{a}=0.04\right)$, SNR0, AL-EG-FDK, and AV-FDK methods. The measured SNR of all
TABLE V

SNR MEASUREd INSIDE THE LEFT VeNTRICLE. The LEFT TABLE PROVIDES A Summary of SNR Measures of All Methods, While the Right Table SPECIFICALLY SHOWS THE SNR DEPENDING ON $\sigma_{a}$ AND $\sigma_{b}$ FOR THE SNR1 AND SNR2 METHOD ON THE ANIMAL MODEL

\begin{tabular}{|c|c|c|c|c|c|}
\hline EG-FDK & 45.65 & $\sigma_{a}$ & $\sigma_{b}$ & SNR1 & SNR2 \\
\hline MC-FDK & 48.98 & 0.005 & 0.5 & 49.44 & 49.40 \\
\hline SNR1 & 63.85 & 0.01 & 4.5 & 54 & 49.58 \\
\hline SNR2 & 63.97 & 0.02 & 12.5 & 60.01 & 51.85 \\
\hline AL-EG-FDK & 49.88 & 0.04 & 28.5 & 63.25 & 60.02 \\
\hline SNR0 & 64.87 & 0.155 & 120.5 & 63.85 & 63.97 \\
\hline AV-FDK & 50.52 & 0.25 & 196.5 & 63.53 & 63.51 \\
\hline
\end{tabular}

methods is presented in Table V. For in vivo data, the MC-FDK and SNR2 methods outperform the EG-FDK method, providing both higher SNR and improved spatial resolution. The impact of the standard deviation parameters $\sigma_{a}$ and $\sigma_{b}$ of the SNR1 and SNR2 methods are shown in Fig. 10 and Table V. The figure shows the resulting resolution at $30 \%$ edge response versus the SNR for the range $\sigma_{a}=0.005,0.01, \ldots, 0.25$ and $\sigma_{b}=0.5,4.5, \ldots, 196.5$. The AL-EG-FDK and AV-FDK methods provide a resolution of about $0.7 \mathrm{lp} / \mathrm{cm}$ and a SNR of about 50. The EG-FDK method has the lowest SNR with a resolution of about $0.9 \mathrm{lp} / \mathrm{cm}$. The SNR1 and SNR2 methods provide measurements ranging from the SNR and resolution of the MC-FDK method to the SNR0 method. The SNR2 method outperforms the SNR1 method, although both provide an advantageous nonlinear regularization for the SNR-resolution trade-off between the MC-FDK and SNR0 method as shown in Fig. 10. The numerical results summarized in Table V are reflected in multiplanar reconstructions (MPR) (intensity window width 621) shown in Fig. 11. Contrast-filled ventricles, as shown in the marked region of interest in the MPR (see black arrow in Fig. 11-AL-EG-FDK), and vessels appear more homogeneous as shown in Fig. 11-SNR1, SNR2, SNR0, and MC-FDK compared to AL-EG-FDK, AV-FDK and EG-FDK. Consistent with the in vitro results, the contrast-filled region of the SNR2 method is more homogeneous than that of SNR1. Especially in regions of strong motion, e.g., the area indicated by the small circle in Fig. 11-AL-EG-FDK, the sharpness of the edge differs between the MC-FDK based methods. For example, the edge inside the black circle is sharper in the MC-FDK method as compared to the EG-FDK. For the SNR2 method, edge sharpness is comparable to that of EG-FDK, while SNR is significantly increased. However, as shown in Fig. 11-SNR1, the edges becomes more blurred as compared to EG-FDK, SNR2, and the MC-FDK method. For the uniform weighting scheme SNR0, the edges become more blurred, while the SNR increases from 45.65 (EG-FDK) to 64.87. A uniform averaging using AL-EG-FDK results in strongly blurred edges such as seen in a nongated reconstruction (Fig. 11-AV-FDK). This emphasizes the performance of MC-FDK based methods using an estimated subject-specific MVF for motion correction.

\section{Computational Complexity}

Initial Reconstruction: The EG-FDK reconstruction of one volume ( $n=256^{3}$ voxels) can be performed in less than $3 \mathrm{~s}$ using GPU acceleration. 


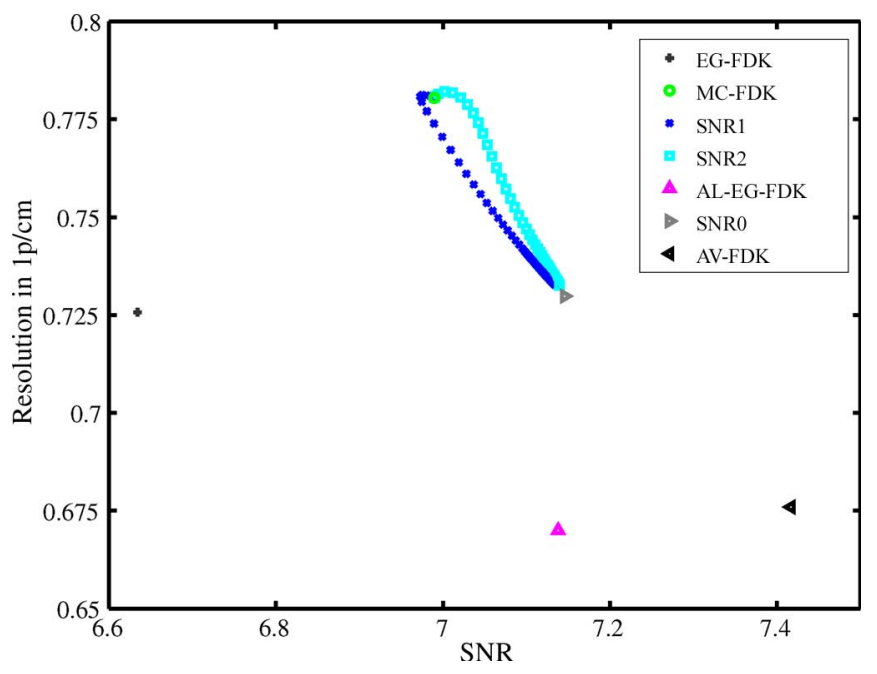

Fig. 9. In vitro phantom: Edge response at $30 \%$ in $1 \mathrm{p} / \mathrm{cm}$ versus SNR.

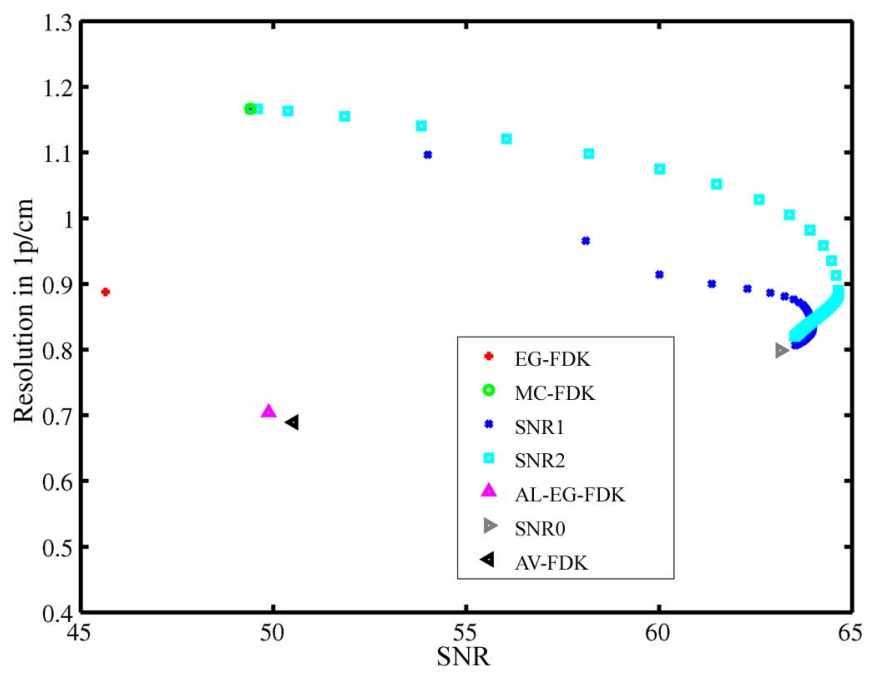

Fig. 10. Edge response at $30 \% \mathrm{in} \mathrm{lp} / \mathrm{cm}$ versus SNR of the animal model. The standard deviation parameters of the SNR1 and SNR2 methods are varying with $\sigma_{a}=0.05, \ldots, 0.25$ and $\sigma_{b}=0.5, \ldots, 196.5$.

Motion Estimation: For the motion estimation, $N_{b}$ initial reconstructions are computed and then $\left(N_{b}-1\right)$ pairwise registrations are required. Using a fast DCT-technique for inversion of the sparse and structured $n \times n$ matrix, a complexity of $O(n \log (n))$ is seen for each iteration of the registration of one volume pair. Most of the work is done on coarse grids and only about five iterations are performed on full resolution. This results in a registration runtime of about 2 min for one pair. The complexity of the MVF interpolation using linear interpolation is $O\left(n\left|P^{t, w}\right|\right)$, since for each projection image one vector field is interpolated.

Reconstruction: The runtime of the MC-FDK method is much higher compared to EG-FDK, because additional computational costs include loading the specific 3-D MVF for each projection image used for the reconstruction and trilinear interpolation of each back-projected image for warping. Using a non runtime optimized $\mathrm{C}++$ implementation, one MC-FDK reconstruction takes about $3 \mathrm{~min}$. For SNR0, SNR1, and SNR2 $N_{b}$
TABLE VII

NOTATION AND ABBREVIATIONS

\begin{tabular}{ll}
\hline$P$ & projection index set \\
$t$ & cardiac phase \\
$t_{i}$ & discrete temporal samples for initial recon. \\
$t_{r}$ & reference cardiac phase \\
$\beta$ & projection image index \\
$\tau$ & function maps $\beta$ to $t$ \\
$\tau_{E}\left(P^{t, w}\right)$ & effective cardiac phase after ECG-gating \\
$\sigma_{C P}^{2}\left(P^{t, w}\right)$ & cardiac phase variance after ECG-gating \\
$w$ & index for gating window \\
$P^{t, w}$ & ECG-gated projection index set \\
$N$ & volume size $N \times N \times N$ \\
$N_{b}$ & number of initial reconstructions \\
$N_{s}$ & number of subsequent C-arm sweeps \\
$\boldsymbol{V}_{t, w}(\boldsymbol{X})$ & Volume of ECG-gated FDK reconstruction \\
$\boldsymbol{f}_{\tilde{\boldsymbol{f}}}$ & 2-D projection image \\
$\tilde{\boldsymbol{F}}_{\beta}(\boldsymbol{X})$ & filtered projection image \\
$\tilde{\boldsymbol{F}}_{\beta}\left(\boldsymbol{X}+\boldsymbol{U}_{\tau(\beta)}\right)$ & filtered backprojected image \\
$\hat{\boldsymbol{V}}_{t_{r}}^{E}\left(\boldsymbol{x}_{j}\right)$ & spatially warped $\tilde{\boldsymbol{F}}_{\beta}(\boldsymbol{X})$ \\
$\boldsymbol{X}$ & SNR enhanced reconstruction \\
$\boldsymbol{x}_{j}$ & grid points of voxel \\
$\boldsymbol{U}$ & lattice point \\
$\boldsymbol{u}_{j}$ & dense deformation field \\
$C_{j}^{t_{r}, w}$ & 3-D displacement vector \\
$M_{j}$ & contribution weight \\
$\mathrm{MRB}$ & normalization function \\
\hline & motion-related blurring \\
\hline
\end{tabular}

Reconstruction Methods

\begin{tabular}{ll}
\hline FDK & Feldkamp reconstruction \\
EG-FDK & ECG-gated FDK reconstruction using subset $P^{t, w=0}$ \\
MC-FDK & ECG-gated, motion compensated FDK-like eqn. (8) \\
MC-FDK-P & 2-D interpolation in projection space in MC-FDK \\
MC-FDK-B & 3-D interpolation in back-projection space in MC-FDK \\
SNR0 & SNR enh. averaging scheme via (9) and $C_{j}^{t_{r}, w}=1$ \\
SNR1 & cardiac phase variance scheme using (9) and (10) \\
SNR2 & intensity scheme using (9) and (12) \\
AV-FDK & non-gated Feldkamp (averaging) using eqn. (14) \\
AL-EG-FDK & averaged non-rigid aligned initial reconstructions (13) \\
GT-FDK & non-moving ground truth reconstruction
\end{tabular}

motion corrected volumes using MC-FDK are reconstructed. The complexity of the weighting schemes SNR1 and SNR2 is then $O\left(n N_{b} C_{c}\right)$, where $C_{c}$ is a constant and depends on the complexity of the weighting function. The weighting takes about a second. The overall estimation plus reconstruction time on a CPU is about $12 \min \left(N_{b}=4, N=256\right)$.

\section{Discussion AND CONCLUSION}

\section{A. Discussion}

1) Dynamic Filtered Backprojection: The results from the animal model and from the in vitro phantom indicate that the voxel-dependent intensity weighting of the SNR2 provides a better trade-off between spatial resolution and SNR than the SNR1 method. For both the animal model and the in vitro phantom, the SNR2 method provided an advantageous nonlinear regularization between SNR and spatial resolution such that a higher SNR can be achieved, while the resolution still remains above values provided by EG-FDK and SNR0. However, results from the in vitro phantom showed that the SNR1 method is more linear in regularizing between resolution and SNR, while for the animal model a more beneficial trade-off 


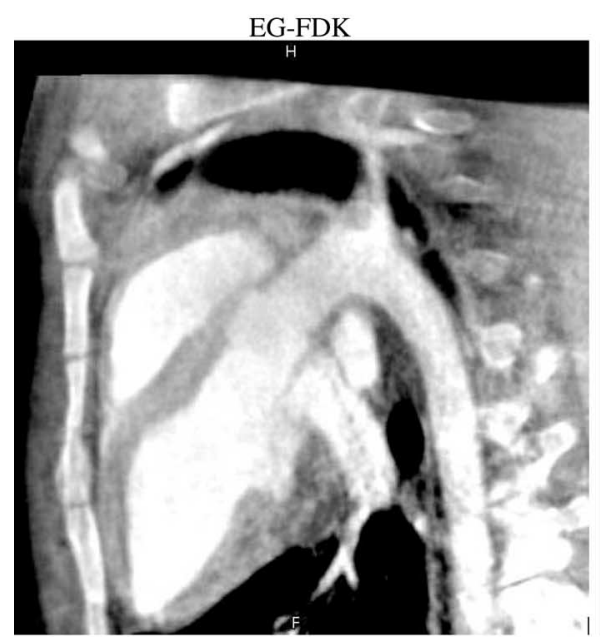

SNR 1
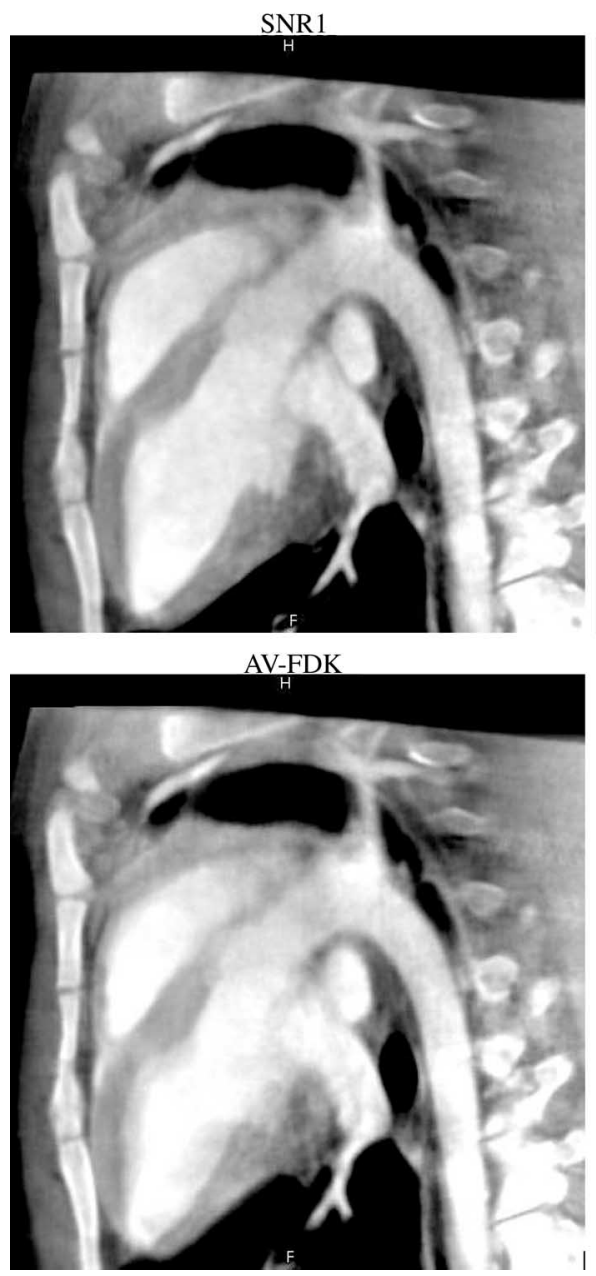

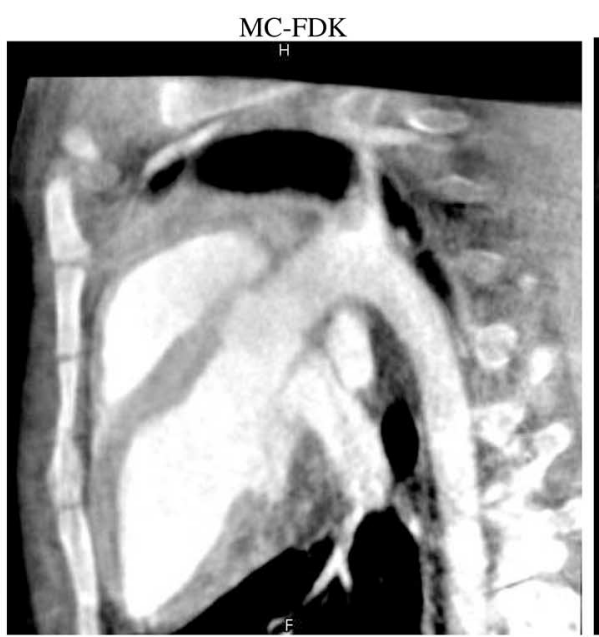

SNR2
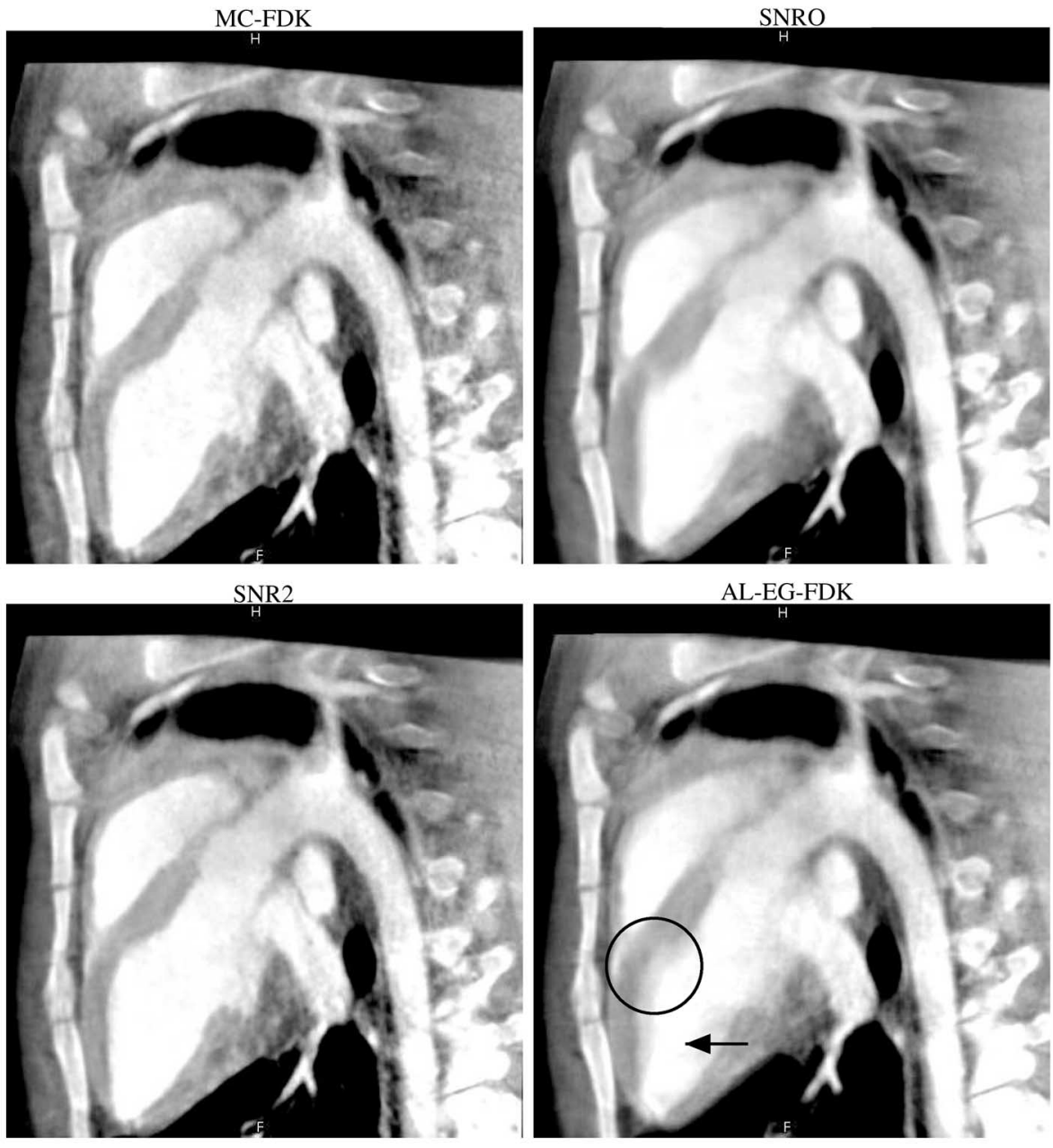

AL-EG-FDK

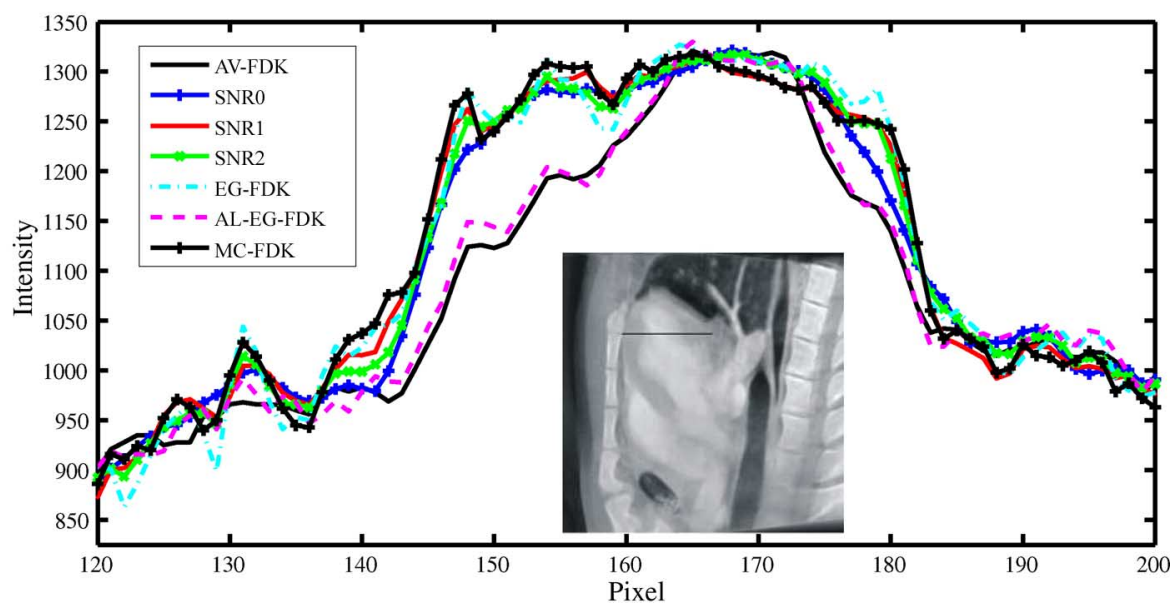

Fig. 11. Multiplanar reconstructions of all seven reconstruction methods of a swine. A measured intensity profile is shown in the bottom right figure. The intensity profile is measured from left to right along the black line as shown in the thumbnail $\left(\sigma_{a}=0.02, \sigma_{b}=100\right)$.

can be achieved (see Figs. 9 and 10). Compared to the MC-FDK method, we observed higher motion blurring in the motion correction methods in which all projections contribute to the final reconstruction (SNR0, SNR1, and SNR2) because motion-corrected filtered backprojections that lie farther outside the targeted cardiac phase window are included. The results also show that the MC-FDK method provides increased edge sharpness compared to EG-FDK and therefore is a reliable reference for the weighting scheme of the SNR2 method. The cardiac phase-based weighting scheme of the SNR1 method also outperforms the uniform weighting scheme of the SNR0 method as shown in Figs. 10 and 9. A performance summary is presented in Table VI. As shown in Figs. 10 and 9, the trade-off between spatial resolution and SNR can be regularized using the Gaussian weighting methods SNR1 and SNR2 such that increased SNR can be gained while the spatial resolution can 


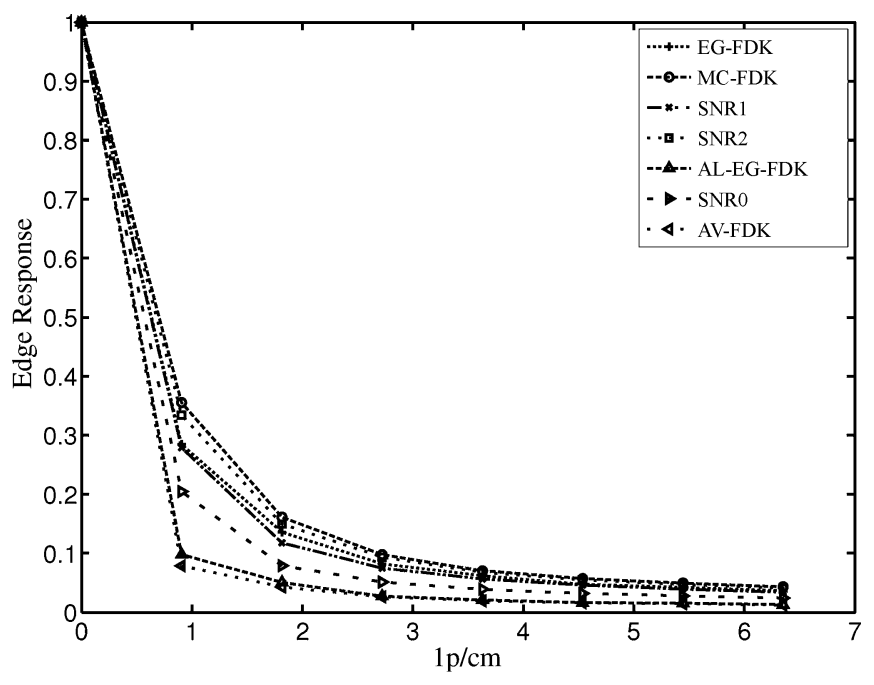

Fig. 12. Edge response functions $\left(\sigma_{1}=0.04, \sigma_{2}=28.5\right)$ of the animal model.

TABLE VI

PERFormance Summary of ReCONSTRUCTION METHODS. THE RANKING IS ORDERED FROM LEFT (BEST) TO RIGHT. (A) EG-FDK, (B) AV-FDK, (C) AL-EG-FDK, (D) MC-FDK, (E) SNR0, (F) SNR1, (G) SNR2

\begin{tabular}{cccccccc}
\hline Complexity & A & B & C & D & E & F & G \\
\hline SNR & G & F & E & B & C & D & A \\
\hline $\mathrm{lp} / \mathrm{cm}(30 \%)$ & D & G & F & A & E & C & B \\
\hline
\end{tabular}

be approximately preserved. In our evaluation, the noise is measured as the variance of a region where a homogeneous attenuation value is expected. It is expected that methods that take into account the same number of projection data provide comparable SNR values. However, in our case the increase of SNR is provided in addition to a reduction of motion artifacts.

2) $A L-E G-F D K$ Versus $M C-F D K$ : The comparison between motion correction during reconstruction (MC-FDK) and retrospective alignment of several cardiac phases (AL-EG-FDK) after ECG-gated FDK reconstruction using the same MVF estimate shows a significant advantage for the MC-FDK algorithm. The key is that the AL-EG-FDK method only aligns anatomical structures and is therefore limited by the initial image quality. AL-EG-FDK produces an average of possible blurred ventricle edges of several cardiac phases and, in the ideal case, the edges are only perfectly aligned and not sharpened. In contrast, increased edge sharpness can be achieved if the FBPs are spatially warped such that, during the accumulation step of MC-FDK, the filtered projection data contribute to the correct spatial edge position and thus to improved edge sharpness.

3) Retrospective Motion Estimation: Although not described in detail here, we have observed that the temporal distribution of the initial reconstructions should uniformly cover the full R-R interval even if those initial reconstructions are blurred. The curvature regularization to enforce smoother spatial deformations is also significant, especially for blurred initial reconstructions correlating with a high cardiac phase variance. We assume that the remaining blurring in ECG-gated reconstructions is compa- rable for different cardiac phases. It is well known that in multilevel image registration the energy minimum is not shifted in its location on coarser resolutions. It can therefore be expected that a MVF can be estimated on a blurred representation of the object like the initial reconstructions using a proper regularization. Empirical studies also showed that while temporal regularization can improve the motion estimate, the improvement was not significant and depended on the object. Temporal regularization affects the spatial smoothness, and thus must be implemented very carefully. Heart motion is nonlinear in time and the temporal sampling of our data is sparse, temporal regularization must incorporate this nonlinearity and should in addition be bounded by the temporal sampling. This is, in practice, a nontrivial task and can result in a less optimal spatial alignment of edges in order to comply with the requirement of temporal smoothness. However, for the motion correction a precise spatial alignment of edge structure is crucial to provide sharp edges in a corrected reconstruction. One solution for a fast temporal regularization is to smooth the temporal trajectory of each voxel using a time dependent Gaussian kernel that is adapted to the heart motion. The pairwise registration is performed in parallel and so the smoothing could be applied during each iteration during minimization.

4) Clinical CT: Although not within the scope of this paper, our approach could also be used with preoperative clinical CT data. Calculation of the MVF could be carried out using CT scans acquired prior to the intervention. After alignment between the intraoperative C-arm CT volume and the clinical CT data, the MVF could be mapped to the $\mathrm{C}$-arm data and used to provide corrected $\mathrm{C}$-arm $\mathrm{CT}$ volume reconstructions. Note that the motion estimation we present should not be compared directly to those approaches that are used for cardiac CT because the cardiac phase variance of C-arm CT ECG-gated projection sets is usually much higher than in clinical cardiac CT.

\section{B. Conclusion}

We conclude that standard 3-D-3-D nonrigid registration, based on initial EG-FDK reconstructions, provides a motion estimate for retrospective motion correction. We demonstrated that by combining several MVFs via cubic-spline interpolation into a 4-D-MVF, retrospective motion correction using the MC-FDK algorithm can be achieved. Compared to the AL-EG-FDK method, where only EG-FDK reconstructions, motion estimation, and regridding of the volumes is required, the SNR0, SNR1, and SNR2 methods are computationally more expensive. The AL-EG-FDK algorithm provides comparable results to nongated Feldkamp (AV-FDK) where strong motion blurring is observed. We also conclude that, for most of the experiments, the MC-FDK method reduces motion related blurring significantly and edge sharpness is maximized. Furthermore, the SNR ratio can be increased by up to $70 \%$ by using all acquired projection images of a multisweep scan using the MC-FDK algorithm in combination with the SNR0, SNR1, or SNR2 method. The SNR1 and SNR2 weighting methods outperform SNR0 by weighting the contribution of motion corrected FBPs based on intensity deviations or based on the cardiac phase variance. In our experiments SNR2 outperformed the SNR1 method. We further conclude that the SNR1 
and SNR2 weighting methods address the trade-off between increased SNR and motion blurring caused by approximate motion estimation and correction methods using an FDK-like algorithm. However, the experiments showed that an increased SNR is obtained at the cost of a slightly higher blurring of edges.

In summary, the introduced motion estimation and correction framework provides increased SNR while reducing motion-related blurring, which may be particularly important in applications that require segmentation such as delineation of contrast- filled ventricles. We also show that our refined motion estimation/correction method can be applied to cardiac C-arm CT data, where cardiac motion is locally less smooth than that of respiratory motion as introduced by $\mathrm{Li}$ et al. [22]. In addition, MC-FDK has high potential for practical application since the newest hardware accelerated FDK-like reconstructions take less than $3 \mathrm{~s}$ for $256^{3}$ volumes and about 200 projections. The motion registration of the volume pairs can be done in parallel and, using fast linear interpolation, the computational cost to compute the MVF as well as the spatial warping of the FBP is acceptable. The framework introduced here has applications beyond cardiac imaging, and may be particularly useful for the estimation and correction of respiratory-motion-related artifacts.

\section{REFERENCES}

[1] L. A. Feldkamp, L. C. Davies, and J. W. Kress, "Practical cone-beam algorithm," J. Opt. Soc. Am., vol. A1, pp. 612-619, 1984.

[2] G. Lauritsch, J. Boese, L. Wigström, H. Kemeth, and R. Fahrig, "Towards cardiac C-arm computed tomography," IEEE Trans. Med. Imag., vol. 25, no. 7, pp. 922-934, Jul. 2006.

[3] D. Schäfer, J. Borgert, V. Rasche, and M. Grass, "Motion-compensated and gated cone beam filtered back-projection for 3-D rotational x-ray angiography," IEEE Trans. Med. Imag., vol. 25, no. 7, pp. 898-906, Jul. 2006.

[4] M. Prümmer, L. Wigstrom, J. Hornegger, J. Boese, G. Lauritsch, N. Strobel, and R. Fahrig, "Cardiac C-arm CT: Efficient motion correction for 4D-FBP," in Nucl. Sci. Symp. Conf. Rec., San Diego, CA, Oct. 2006, vol. 4, pp. 2620-2628, 10.1109/NSSMIC.2006.354444.

[5] M. Prümmer, R. Fahrig, L. Wigström, J. Boese, G. Lauritsch, N. Strobel, and J. Hornegger, "Cardiac C-arm CT: 4-D non-model based heart motion estimation and its application," Proc. SPIE Med. Imag.: Phys. Med. Imag., vol. 6510, p. 651015, 2007.

[6] T. Li, E. Schreibmann, B. Thorndyke, G. Tillman, A. Boyer, A. Koong, K. Goodman, and L. Xing, "Radiation dose reduction in four-dimensional computed tomography," Med. Phys., vol. 32, no. 12, pp. 3650-3660, Dec. 2005.
[7] T. Li, E. Schreibmann, Y. Yang, and L. Xing, "Motion correction for improved target localization with on-board cone-beam computed tomography," Phys. Med. Biol., vol. 51, no. 2, pp. 253-267, 2006.

[8] A. F. Frangi, W. J. Niessen, and M. A. Viergever, "Three-dimensional modeling for functional analysis of cardiac images: A review," IEEE Trans. Med. Img., vol. 20, no. 1, pp. 1-25, Jan. 2001.

[9] K. Taguchi, W. P. Segars, H. Kudo, E. C. Frey, and E. K. Fishman, "Toward time resolved cardiac CT images with patient dose reduction: Estimating the global heart motion," Phys. Med. Imag., vol. 6142, no. 1.

[10] K. Taguchi, W. P. Segars, H. Kudo, E. C. Frey, E. K. Fishman, and B. M. W. Tsui, "Toward time resolved cardiac CT images with patient dose reduction: Image-based motion estimation," in Proc. IEEE NSS-MIC Conf., San Diego, CA, 2006, vol. 4, pp. 2029-2032.

[11] D. Shen, H. Sundar, Z. Xue, Y. Fan, and H. Litt, "Consistent estimation of cardiac motions by 4-D image registration," presented at the MICCAI Conf., Palm Springs, CA, Oct. 26-29, 2005.

[12] C. Blondel, G. Malandain, R. Vaillant, and N. Ayache, "Reconstruction of coronary arteries from a single rotational X-ray projection sequence," IEEE Trans. Med. Imag., vol. 25, no. 5, pp. 653-663, May 2006, 0278-0062.

[13] J. Modersitzki, Numerical Methods for Image Registration. Oxford, U.K.: Oxford Univ. Press, 2004.

[14] D. L. Parker, "Optimal short scan convolution reconstruction for fanbeam CT,” Med. Phys., vol. 9, no. 2, pp. 254-257, Apr. 1982.

[15] T. Rodet, F. Noo, and M. Defrise, "The cone-beam algorithm of Feldkamp, Davis, and Kress preserves oblique line integrals," Med. Phys., vol. 31, no. 7, pp. 1972-1975, 2004.

[16] T. Brox, R. van den Boomgaard, F. B. Lauze, J. van de Weijer, J. Weickert, P. Mrázek, and P. Kornprobst, "Adaptive structure tensors and their applications," in Visualization and Processing of Tensor Fields. New York: Springer, Jan. 2006, pp. 17-47.

[17] InSpace [Online]. Available: http://www.insideinspace.com Jan. 2007

[18] H. H. Barrett, K. J. Myers, and S. Rathee, "Foundations of image science," Med. Phys., vol. 31, no. 4, pp. 953-953, 2004.

[19] T. M. Lehmann, C. Gonner, and K. Spitzer, "Survey: Interpolation methods in medical image processing," IEEE Trans. Med. Imag., vol. 18, no. 11, pp. 1049-1075, Nov. 1999.

[20] J. Han, B. Berkels, M. Droske, J. Hornegger, M. Rumpf, C. Schaller, J. Scorzin, and H. Urbach, "Mumford-shah model for one-to-one edge matching," IEEE Trans. Image Process., vol. 16, no. 11, pp. 2720-2732, Nov. 2007.

[21] G. Hermosillo, "Variational methods for multimodal image matching," Ph.D. dissertation, Univ. Nice, INRIA, Nice, France, 2002.

[22] T. Li, A. Koong, and L. Xing, "Enhanced 4-D cone-beam CT with inter-phase motion model," Med. Phys., vol. 34, no. 9, pp. 3688-3695, 2007, 17926972.

[23] L. Desbat, S. Roux, and P. Grangeat, "Compensation of some time dependent deformations in tomography," IEEE Trans. Med. Imag., vol. 26, no. 2, pp. 261-269, Feb. 2007.

[24] K. Taguchi and H. Kudo, "Motion compensated fan-beam reconstruction for computed tomography using derivative backprojection filtering approach," presented at the 9th Int. Meeting Fully Three-Dimensional Image Reconstruction Radiol. Nucl. Med., Lindau, Germany, Jul. 9-13, 2007. 